\title{
Preexisting endothelial cells mediate cardiac neovascularization after injury
}

\author{
Lingjuan He, ${ }^{1,2}$ Xiuzhen Huang, ${ }^{1,2}$ Onur Kanisicak, ${ }^{3}$ Yi Li, ${ }^{1,2}$ Yue Wang, ${ }^{1,2}$ Yan Li, ${ }^{1,2}$ Wenjuan Pu, ${ }^{1,2}$ Qiaozhen Liu, ${ }^{1,2}$ Hui Zhang, ${ }^{1,2}$ \\ Xueying Tian, ${ }^{1,2}$ Huan Zhao, ${ }^{1,2}$ Xiuxiu Liu, ${ }^{1,2}$ Shaohua Zhang, ${ }^{1,2}$ Yu Nie, ${ }^{4}$ Shengshou Hu, ${ }^{4}$ Xiang Miao, ${ }^{5}$ Qing-Dong Wang, ${ }^{6}$ \\ Fengchao Wang, ${ }^{7}$ Ting Chen, ${ }^{7}$ Qingbo Xu, ${ }^{8}$ Kathy O. Lui, ${ }^{9}$ Jeffery D. Molkentin, ${ }^{3}$ and Bin Zhou ${ }^{1,2,10,11}$ \\ 'The State Key Laboratory of Cell Biology, Chinese Academy of Sciences (CAS) Center for Excellence in Molecular Cell Science, Shanghai Institute of Biochemistry and Cell Biology, ${ }^{2}$ Key Laboratory of \\ Nutrition and Metabolism, Institute for Nutritional Sciences, Shanghai Institutes for Biological Sciences, CAS, University of Chinese Academy of Sciences, Shanghai, China. ${ }^{3}$ Department of Pediatrics and \\ Molecular Cardiovascular Biology, Cincinnati Children's Hospital Medical Center, Cincinnati, Ohio, USA. ${ }^{4}$ State Key Laboratory of Cardiovascular Disease, Fuwai Hospital, National Center for Cardiovascular \\ Disease, Chinese Academy of Medical Sciences and Peking Union Medical College, Beijing, China. ${ }^{5}$ Flow Cytometry Core Facility, Institute of Health Sciences, Shanghai Institutes for Biological Sciences, \\ CAS, Shanghai, China. ${ }^{6}$ Cardiovascular and Metabolic Diseases, Innovative Medicines and Early Clinical Development Biotech Unit, AstraZeneca, Mölndal, Sweden. ${ }^{7}$ National Institute of Biological Sciences, \\ Beijing, China. ${ }^{8}$ Cardiovascular Division, British Heart Foundation Centre, King's College London, London, United Kingdom. ${ }^{9}$ Department of Chemical Pathology, Li Ka Shing Institute of Health Sciences, The \\ Chinese University of Hong Kong, Prince of Wales Hospital, Shatin, Hong Kong, China. ${ }^{10} \mathrm{Key}$ Laboratory of Regenerative Medicine of Ministry of Education, Institute of Aging and Regenerative Medicine, Jinan \\ University, Guangzhou, China. "School of Life Science and Technology, ShanghaiTech University, Shanghai, China.
}

\begin{abstract}
The mechanisms that promote the generation of new coronary vasculature during cardiac homeostasis and after injury remain a fundamental and clinically important area of study in the cardiovascular field. Recently, it was reported that mesenchymal-to-endothelial transition (MEndoT) contributes to substantial numbers of coronary endothelial cells after myocardial infarction. Therefore, the MEndoT has been proposed as a paradigm mediating neovascularization and is considered a promising therapeutic target in cardiac regeneration. Here, we show that preexisting endothelial cells mainly beget new coronary vessels in the adult mouse heart, with essentially no contribution from other cell sources through cell-lineage transdifferentiation. Genetic-lineage tracing revealed that cardiac fibroblasts expand substantially after injury, but do not contribute to the formation of new coronary blood vessels, indicating no contribution of MEndoT to neovascularization. Moreover, genetic-lineage tracing with a pulse-chase labeling strategy also showed that essentially all new coronary vessels in the injured heart are derived from preexisting endothelial cells, but not from other cell lineages. These data indicate that therapeutic strategies for inducing neovascularization should not be based on targeting presumptive lineage transdifferentiation such as MEndoT. Instead, preexisting endothelial cells appear more likely to be the therapeutic target for promoting neovascularization and driving heart regeneration after injury.
\end{abstract}

\section{Introduction}

Coronary artery disease, manifested by acute myocardial infarction and, subsequently, end-stage heart failure, is the leading cause of mortality worldwide (1). Rapid regeneration of new coronary blood vessels is essential for prolonging survival of the injured myocardium (2). Unraveling new sources of vascular endothelial cells in the heart provides important insights into therapeutic deployment for cardiac regeneration (3). One paradigm in the field for promoting neovascularization is generation of new blood vessels through lineage conversion from other types of differentiated cells (4). Fibroblasts are regarded as terminally differentiated cells, and excessive new fibroblasts

Related Commentary: p. 2892

Conflict of interest: The authors have declared that no conflict of interest exists Submitted: March 7, 2017; Accepted: April 20, 2017.

Reference information: J Clin Invest. 2017;127(8):2968-2981.

https://doi.org/10.1172/JCI93868. are accumulated after tissue injury, such as myocardial infarction $(5,6)$. With the advent of reprogramming technology that introduces exogenous transcription factors, these differentiated fibroblasts could be reprogrammed into functional endothelial cells in vitro, which are capable of mediating neovascularization in tissue-engineered vessels and ischemic tissues $(7,8)$. Whether endogenous fibroblasts or mesenchymal stromal cells (MSCs) contribute to endothelial cells after tissue injury remains enigmatic. Recently, it has been reported that resident fibroblasts generate a substantial number of endothelial cells in the heart after injury (9). In this study, approximately $30 \%-40 \%$ fibroblasts contribute to new coronary endothelial cells in the injured myocardium (9). This in vivo cell-lineage conversion facilitates cardiac repair in mouse heart injury models, and the cell plasticity of fibroblasts may represent a novel therapeutic target for cardiac repair and regeneration (10). The cell-lineage conversion or fate-switch model for new blood vessel formation represents an important paradigm for the potential therapeutic target for enhancing cardiac neovascularization. 
A

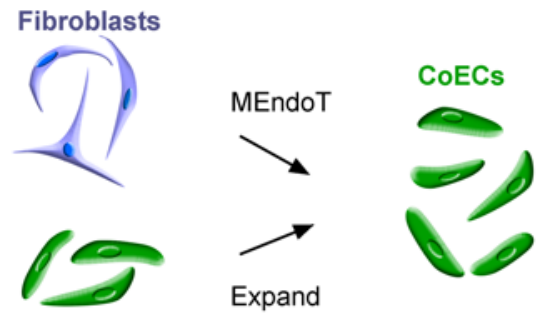

CoECs
B Before injury

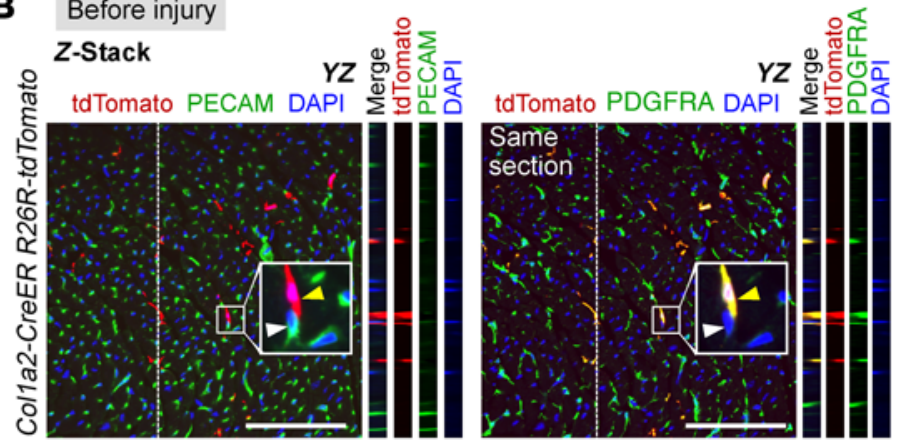

C After injury

tdTomato PECAM DAPI YZ 退음

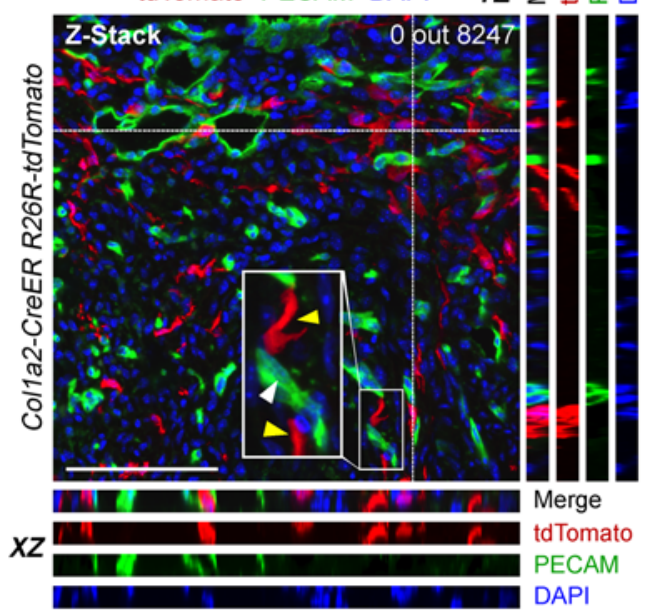

E After injury

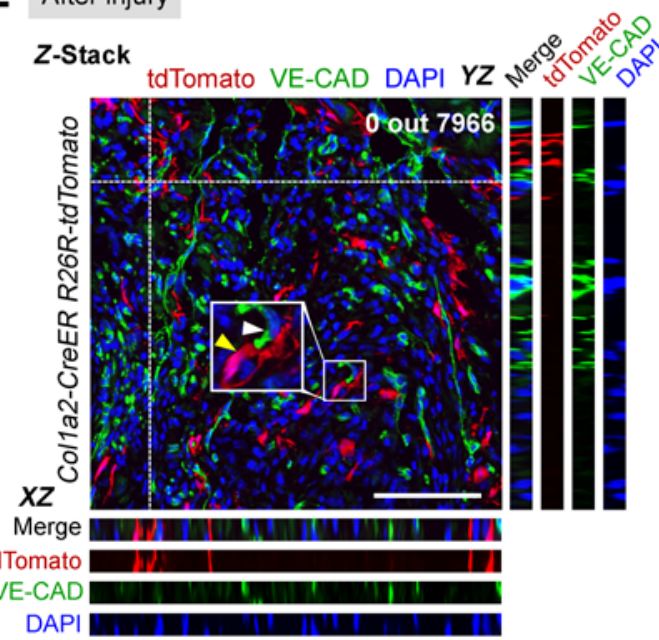

tdTomato PDGFRA DAPI YZ $\sum^{2} \frac{0}{2}$

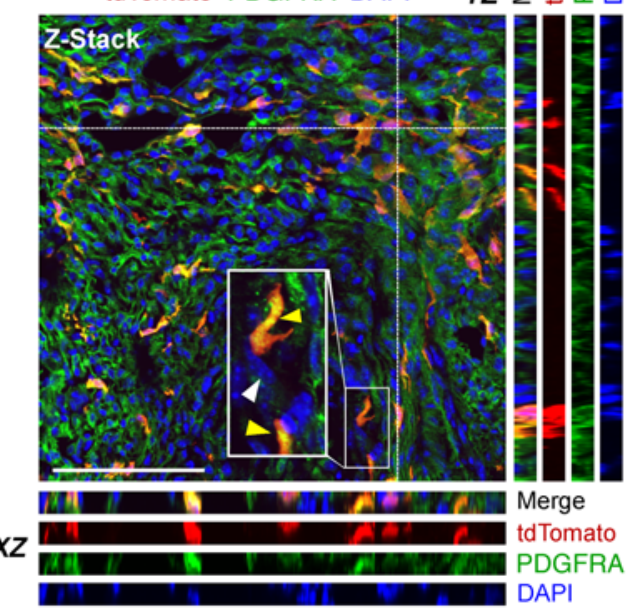

$\mathbf{F}$

After injury
tdTomato VEGFR2 DAPI

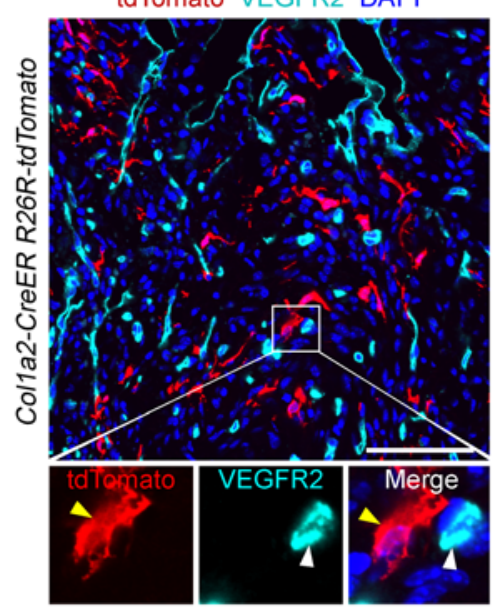

D

FACS analysis
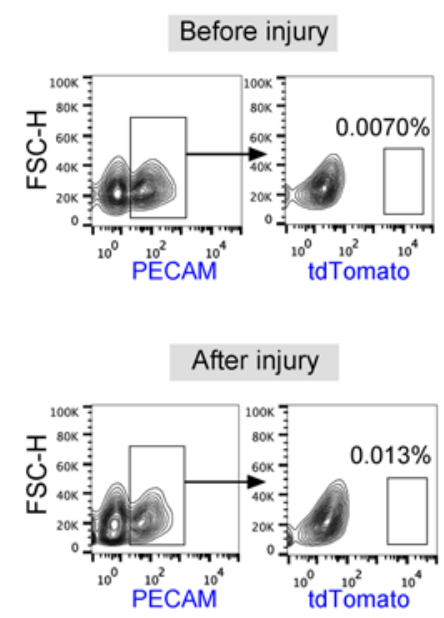

tdTomato perfused-Lectin DAPI

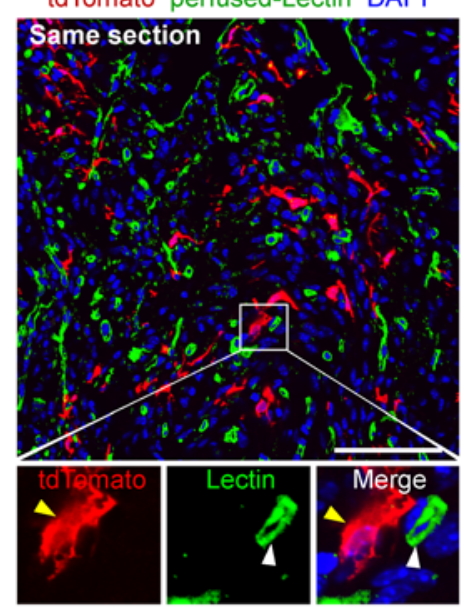

Figure 1. COL1A2+ fibroblasts do not adopt the endothelial cell fate after injury. (A) Models explaining new vessel sources: MEndoT and self-expansion. CoECs, coronary endothelial cells. (B) Z-stack confocal images of heart sections stained for tdTomato, PECAM, and PDGFRA. Col1a2-CreER R26R-tdTomato mice were treated with tamoxifen 2 weeks before analysis. $Y Z$ indicates signals from dotted lines on $Z$-stack images. Yellow arrowheads indicate PDCFRA+tdTomato fibroblasts; white arrowheads indicate PECAM+tdTomato- endothelial cells. (C) Immunostaining for tdTomato, PDCFRA, and PECAM on sections of postinjury hearts. tdTomato+ cells express fibroblast marker PDGFRA+ (yellow arrowheads), but not endothelial cell marker PECAM (white arrowheads). (D) Flow cytometric analysis of percentage of PECAM+ endothelial cells labeled by Col1a2-CreER transgene (tdTomato+). (E) Immunostaining for VE-CAD and tdTomato on sections of injured heart. tdTomato+ cells (yellow arrowheads) are close to, but were not identified as VE-CAD+ endothelial cells (white arrowheads) in the injured heart. (F) Immunostaining for VEGFR2 and tdTomato on heart sections perfused with FITC-labeled BS1 lectin. tdTomato ${ }^{+}$cells (yellow arrowheads) are not VEGFR2 $2^{+}$lectin ${ }^{+}$vascular endothelial cells (white arrowheads). Scale bars: $100 \mu \mathrm{m}$. Each image is representative of 4 individual hearts. 
During the process of cardiac repair, cardiac fibroblasts are key players and their proliferation is often accompanied by recruitment of blood vascular endothelial cells $(6,11-13)$. Ubil et al. showed that a substantial number of cardiac fibroblasts contribute to coronary vessels through mesenchymal-to-endothelial transition (MEndoT) in the injured heart (9). This surprising lineage conversion, if correct, may shed light on development of a potential therapeutic target for enhancing cardiac neovascularization, exerting broad impact in the field of vascular biology and cardiac regeneration. However, previous studies using other genetic tools demonstrate that resident fibroblast lineages mainly mediate cardiac fibrosis $(5,6)$, suggesting that most fibroblasts adopt fibroblast cell fate after cardiac injury. The substantial contribution of fibroblasts to coronary endothelial cells in the infarcted myocardium $(-30 \%-40 \%$ of fibroblasts) is in contradiction to previous studies $(5,6)$. Since the MEndoT findings are highly influential in the cardiovascular field, presenting a new paradigm for therapeutic neovascularization $(9,10)$, we believe that it is important to reassess this MEndoT process and the contribution of fibroblasts to coronary vessels if conflicting data exist. Here, we generated new genetic tools that specifically targeted these different cell lineages, with the aim of systematically addressing whether and to what extent the cell transdifferentiation process (such as MEndoT) or self-expansion of coronary endothelial cells contributes to cardiac neovascularization after injury (Figure 1A).

\section{Results}

COL1A2 $2^{+}$fibroblasts labeled by transgene Cre do not contribute to coronary endothelial cells. We first established a myocardial ischemia-reperfusion (IR) injury model (9). By Sirius red staining of serial sections from IR hearts, we found significant fibrosis after IR injury (Supplemental Figure 1; supplemental material available online with this article; https://doi.org/10.1172/ JCI93868DS1), validating the success of our injury model. To test whether preexisting fibroblasts contributed to new coronary endothelial cells, we used the Col1a2-CreER (where Col1a2 indicates collagen type I $\alpha 2$ chain) transgene line used in the original report (9). COL1A2 is a fibroblast marker, and the Col1a2-CreER transgene has been used previously to mark fibroblasts $(14,15)$. We crossed the Colla2-CreER line with the same reporter line used in the original report, R26R-tdTomato (16), to trace the fate of fibroblasts. In addition, we adopted the same strategy for tamoxifen treatment and analyzed results at the same time points as previously described (9). Tamoxifen-induced CreloxP recombination removes loxP flanked transcriptional stop cassette, irreversibly marking COL1A2 ${ }^{+}$fibroblasts and all their descendants by tdTomato. As genetic labeling is permanent and heritable, we could detect tdTomato ${ }^{+}$endothelial cells in injured heart when prelabeled COL1A2 ${ }^{+}$fibroblasts gave rise to coronary endothelial cells. Prior to injury induction, Col1a2-CreER labeled $\mathrm{PDGFRA}^{+}$fibroblasts but not $\mathrm{PECAM}^{+}$endothelial cells (Figure 1B). To determine whether MEndoT contributes substantially to blood vessels after injury, we performed fate-mapping studies on Col1a2-CreER R26R-tdTomato heart after IR injury. Z-stack confocal images of injured heart sections showed that tdTomato ${ }^{+}$ cells did not express the endothelial cell marker PECAM (0 out of 8,247 td $^{\text {Tomato }}{ }^{+}$cells counted, $n=4$ ), but still expressed the fibroblast marker PDGFRA (Figure 1C). Our flow cytometric analysis showed that very rare tdTomato ${ }^{+}$cells were found in the PECAM ${ }^{+}$ endothelial cell population before and after injury $(n=4$, Figure 1D). One reasonable explanation for the presence of rare tdTomato signal could be autofluorescent background. Additionally, costaining for tdTomato and VE-cadherin (VE-CAD) showed that there were no tdTomato ${ }^{+} \mathrm{VE}-\mathrm{CAD}^{+}$endothelial cells in the injured heart ( 0 out of 7,966 td Tomato ${ }^{+}$cells counted, Figure $1 \mathrm{E}$ ). We also systematically injected the fluorescent-labeled Bandeiraea simplicifolia (BS1) lectin into the Col1a2-CreER R26R-tdTomato mice before sacrifice and then performed immunostaining on heart sections for tdTomato and VEGFR2. The prelabeled tdTomato ${ }^{+}$ fibroblasts did not adopt the VEGFR2 ${ }^{+}$or lectin $^{+}$endothelial cell fate (Figure $1 \mathrm{~F}$ ). Collectively, our results contradicted the previous lineage-tracing experiments showing that approximately $30 \%-40 \%$ of labeled fibroblasts were found to adopt an endothelial cell fate (9), raising concerns about the therapeutic efficacy of MEndoT in driving heart regeneration after injury.

COL1A2 $2^{+}$fibroblasts labeled by knockin Cre do not contribute to coronary endothelial cells. While we failed to repeat the MEndoT process, it remained possible that the Col1a2-CreER transgene used in our study may not be exactly the same as that in the original study, possibly due to silence of transgene or copy number loss after multiple passages. To address the MEndoT by Col1a 2 endogenous promoter, we generated Col1a2-2A-CreER knockin allele, in which CreER (17) was targeted into the translational stop codon of Colla 2 and linked with the endogenous Col1a2 transcript by a short sequence that encodes self-cleaving $2 \mathrm{~A}$ peptide (Figure $2 \mathrm{~A}$ ). To determine the fate of fibroblasts before and after injury, we crossed Col1a2-2A-CreER with the R26R-tdTomato reporter line (16) to trace COL1A2 ${ }^{+}$fibroblasts. To trace whether these fibroblasts generated endothelial cells, we treated mice with tamoxifen and collected hearts for analysis. Col1a2-2A-CreER-labeled cells expanded significantly after injury (Figure 2, B and C). Prior to injury induction, Col1a2-2A-CreER labeled PDGFRA ${ }^{+}$fibroblasts, but not the $\mathrm{PECAM}^{+}$or VE-CAD ${ }^{+}$endothelial cells (Figure 2, D and E, before injury). To determine whether MEndoT contributed substantially to blood vessels after injury, we performed fate-mapping studies on Col1a2-2A-CreER R26R-tdTomato hearts after IR injury. Z-stack confocal images of injured heart sections showed that tdTomato ${ }^{+}$ cells did not express the endothelial cell marker PECAM (0 out of 6,384 tdTomato $^{+}$cells counted, $n=4$ ), but still expressed the fibroblast marker PDGFRA in the border zone, infarcted region, and remote regions, respectively (Figure 2, D and F, after injury and Supplemental Figure 2, A and C). Similarly, costaining for tdTomato and VE-CAD confirmed that there was no tdTomato ${ }^{+} \mathrm{VE}-\mathrm{CAD}^{+}$ endothelial cell found in the injured heart ( 0 out of 6,947 tdTomato $^{+}$cells counted, Figure 2, E and F, and Supplemental Figure 2, $\mathrm{B}$ and D). We also costained sections with tdTomato and another fibroblast marker, DDR2 (9), and found that most tdTomato ${ }^{+}$cells were DDR2 ${ }^{+}$(Figure 2G), indicating the maintenance of fibroblast cell fate after injury. Our flow cytometric analysis showed that tdTomato ${ }^{+}$cells did not contribute to new $\mathrm{PECAM}^{+}$endothelial cells after injury $(n=4$, Figure $2 \mathrm{H})$. Collectively, these lineage-tracing results contradicted the previous conclusion that fibroblasts contribute to a substantial number of endothelial cells after injury (9), raising concerns about the therapeutic efficacy of MEndoT in driving heart regeneration after injury. 
A

Col1a2-2A-CreER knockin strategy

Col1a2 locus

$$
52^{\text {nd }} \text { exon 2A-CreER-pA 3' UTR }
$$

Targeting vector

Targeted allele

$$
\text { I Homologous }
$$

- $52^{\text {nd }}$ exon 2A-CreER-pA 3 ' UTR
B

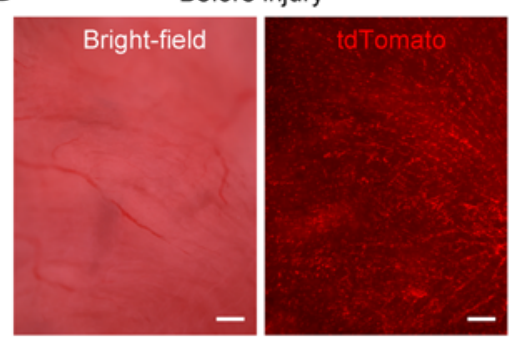

C

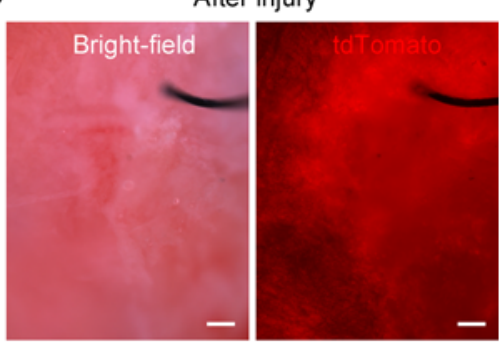

D
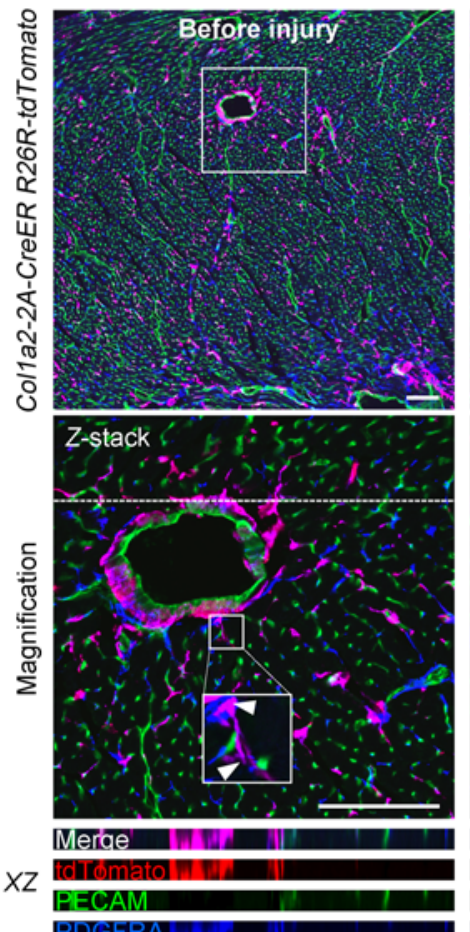

$X Z$

$\mathbf{F}$

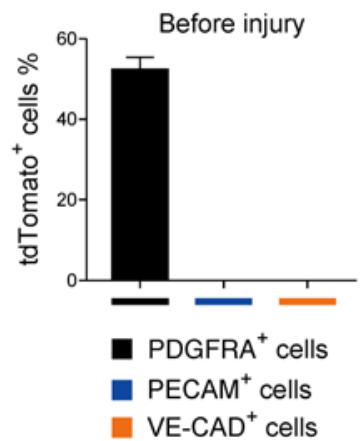

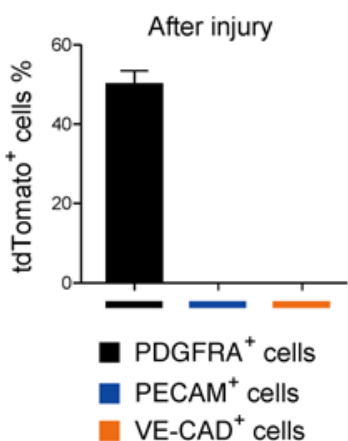

E

tdTomato VE-CAD PDGFRA
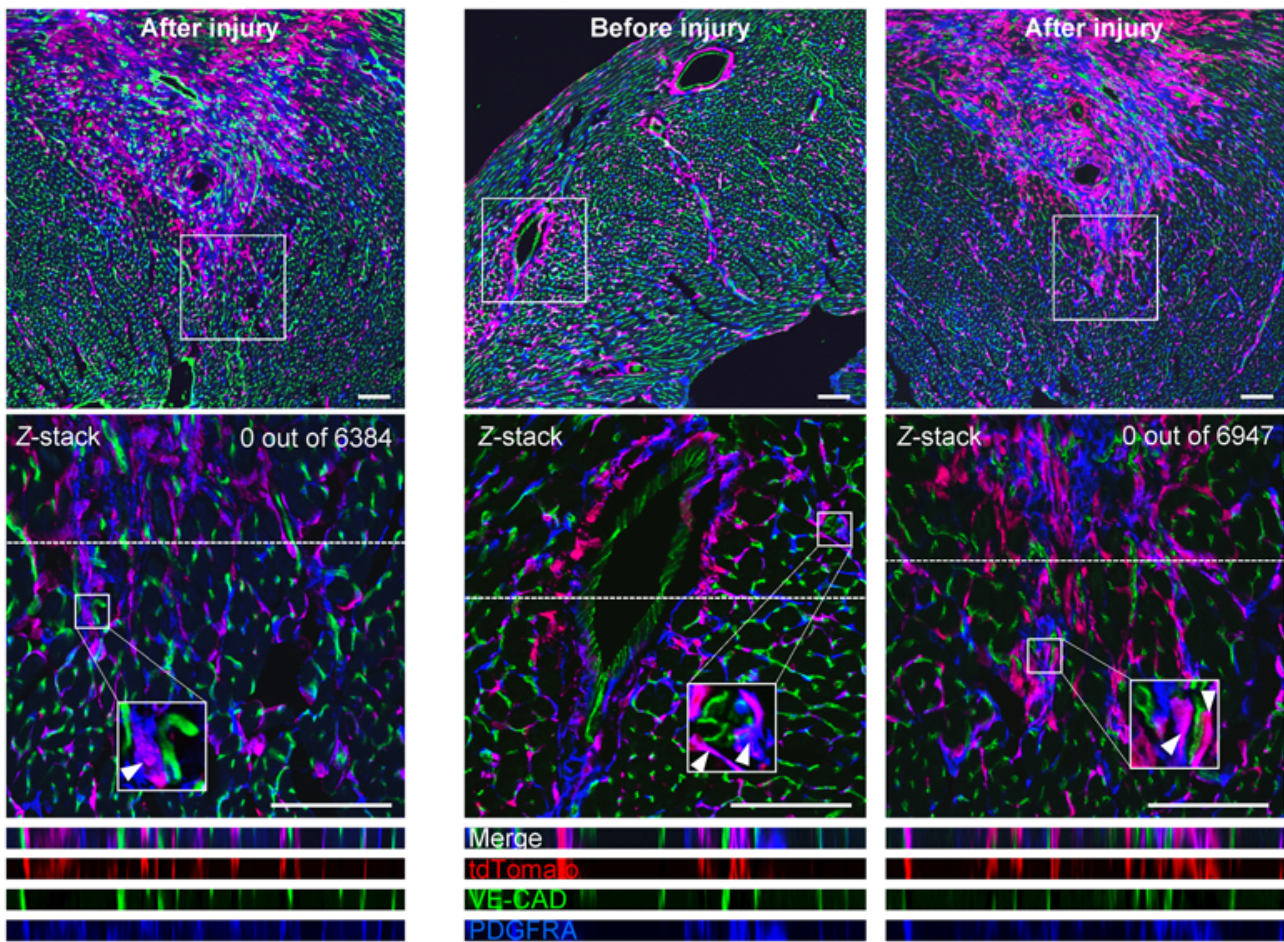

$\mathbf{G}$

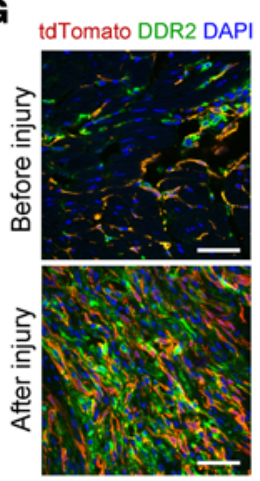

$\mathbf{H}$

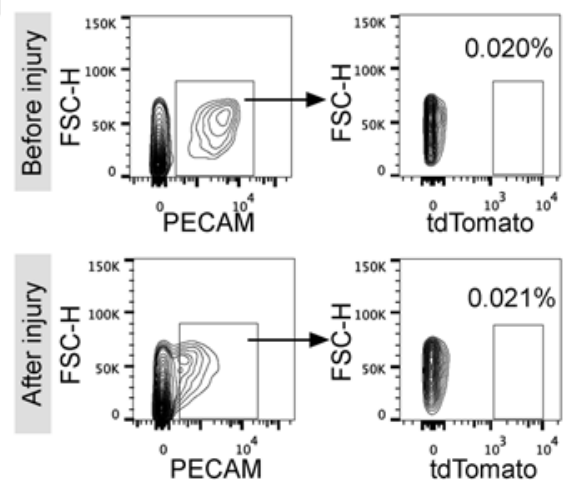

Figure 2. COL1A2 ${ }^{+}$fibroblasts do not contribute to coronary endothelial cells after cardiac injury. (A) Schematic figure showing strategy for generation of Col1a2-2A-CreER allele. (B and C) Whole-mount fluorescence images showing tdTomato in hearts before or after injury. (D and E) Immunostaining for tdTomato, PDGFRA, and PECAM (D) or VE-CAD (E) on sections of injured heart. Boxed regions are magnified in bottom panels. Arrowheads point to tdTomato+PDCFRA ${ }^{+}$PECAM ${ }^{-}$(D) or tdTomato+PDGFRA ${ }^{+} V E-C A D^{-}$cells (E). XZ indicates signals from dotted line in Z-stack images. (F) Quantification of the percentage of tdTomato ${ }^{+}$cells in different lineages (PDGFRA ${ }^{+}, \mathrm{PECAM}^{+}$, or VE-CAD+ cell populations). Data are represented as mean $\pm S E M$. $n=4$. (C) Immunostaining for tdTomato and DDR2 on heart sections before or after injury. (H) Flow cytometric analysis of the percentage of tdTomato endothelial cells. FSC-H, forward scatter-height. Scale bars: $200 \mu \mathrm{m}$ (B, C); $100 \mu \mathrm{m}$ (D, E, C). 
A

Pdgfra-DreER knockin strategy

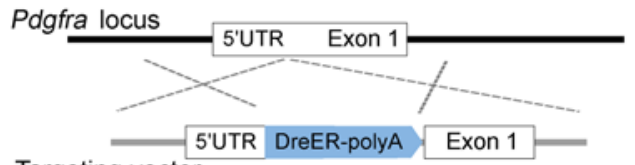

Targeting vector $\quad \downarrow \begin{gathered}\text { Homologous } \\ \text { recombination }\end{gathered}$

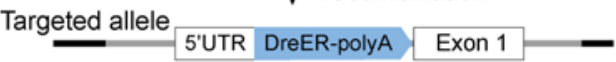

B

Lineage tracing strategy

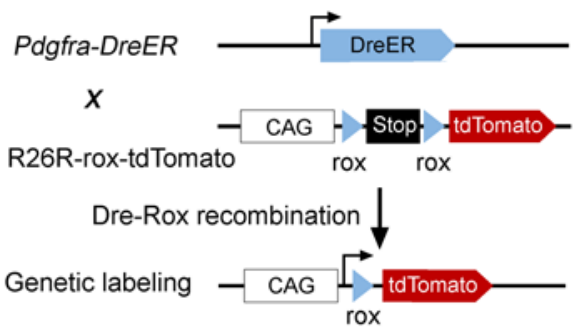

C

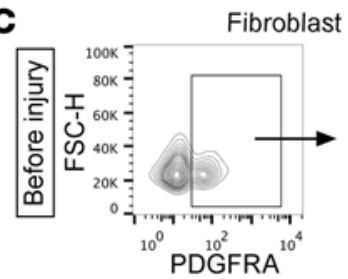

D
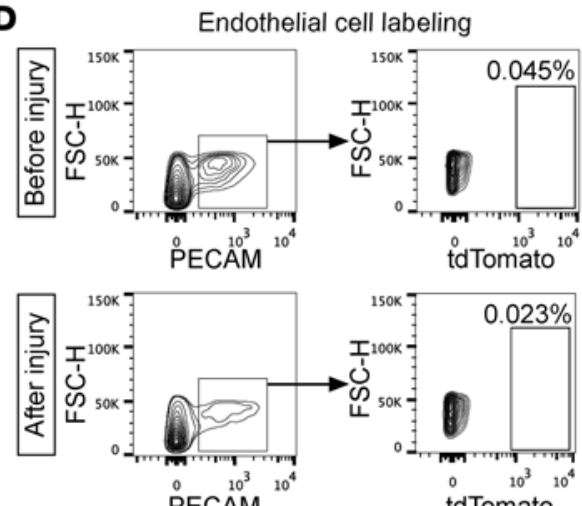

PECAM

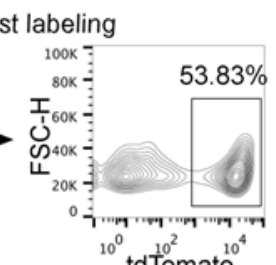

tdTomato

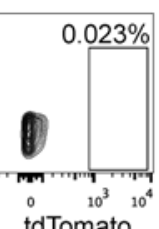

E tdTomato
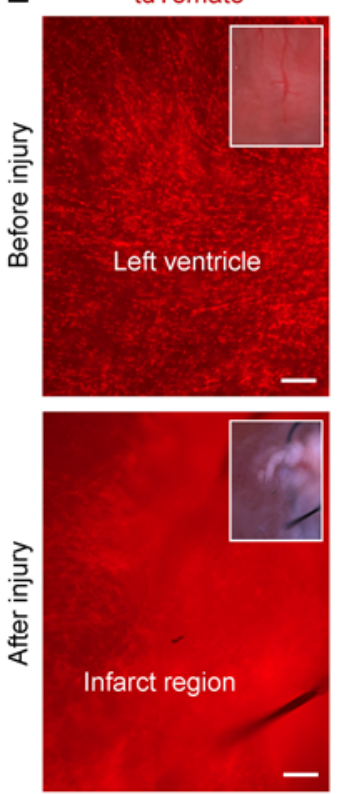

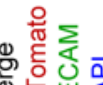

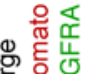

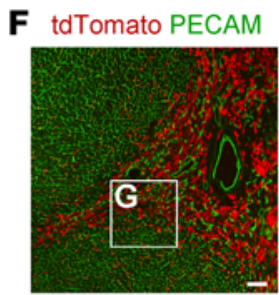

tdTomato VE-CAD

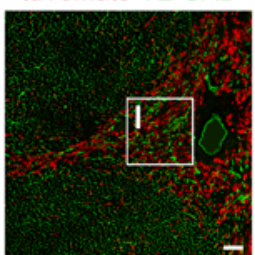

dTomato PDGFRA

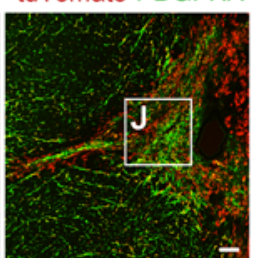

$\mathbf{K}$

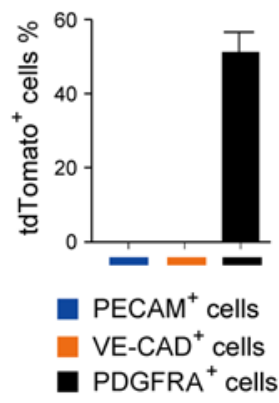

G

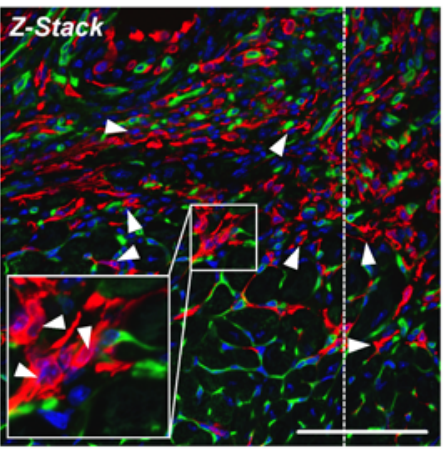

I

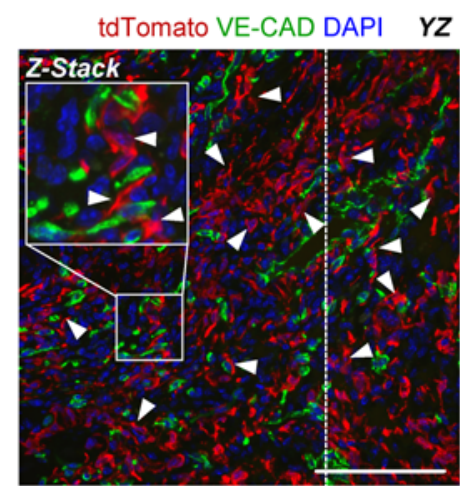

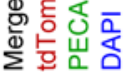

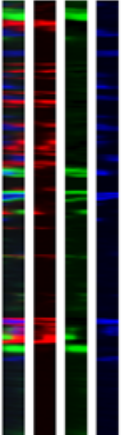

H tdTomato PDGFRA DAPI

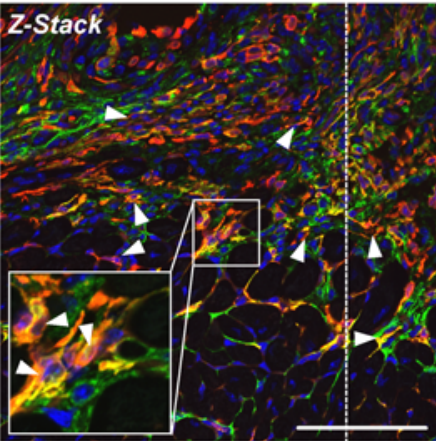

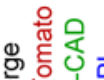

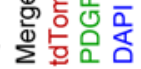

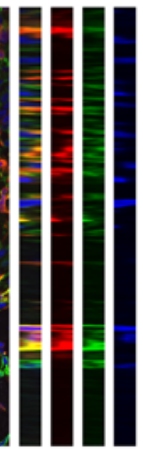

帝它岁京

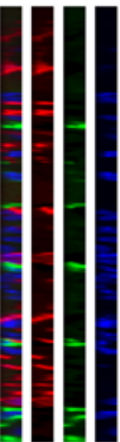

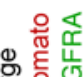

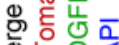
J tdTomato PDGFRA DAPI $\quad Y Z \sum_{2}^{\infty} \frac{0}{0}$

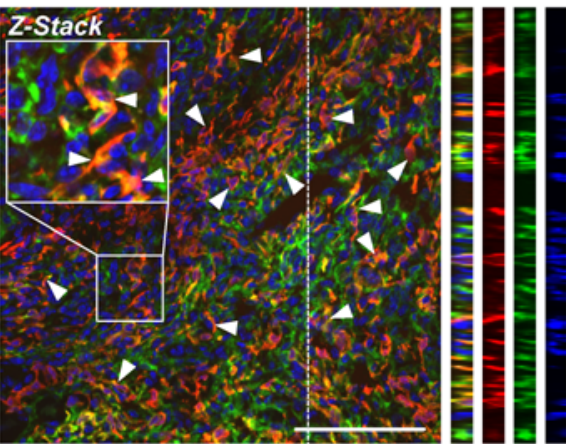

Figure 3. PDGFRA+ fibroblasts do not contribute to coronary endothelial cells after cardiac injury. (A) Schematic figure showing strategy for generation of Pdgfra-DreER allele. (B) Schematic figure showing genetic-lineage-tracing strategy for PDCFRA ${ }^{+}$cells by Dre-rox recombination. (C and $\left.\mathbf{D}\right)$ Flow cytometric analysis of the percentage of tdTomato fibroblasts (C) or tdTomato endothelial cells (D). (E) Whole-mount fluorescence image showing tdTomato in hearts before and after injury. (F-J) Immunostaining for tdTomato, PDGFRA, PECAM, or VE-CAD on sections of injured heart. Boxed regions in $\mathbf{F}$ are magnified in G-J. Arrowheads point to tdTomato+PDCFRA+PECAM- (G, H) or tdTomato+PDGFRA+VE-CAD- cells (I and J). YZ indicates signals from dotted line in Z-stack images in G-J. (K) Quantification of the percentage of tdTomato cells in different lineages (PECAM+, VE-CAD+, or PDCFRA ${ }^{+}$cell populations). Data are represented as mean \pm SEM. $n=4$. Scale bars: $200 \mu \mathrm{m}(\mathbf{E}) ; 100 \mu \mathrm{m}$ in $(\mathbf{F}-\mathrm{J})$. 
PDGFRA fibroblasts do not contribute to new coronary endothelial cells. We next employed the widely used fibroblast marker PDGFRA to drive inducible recombinase for lineage tracing. We inserted a cDNA encoding Dre and strogen receptor (ER) fusion protein into the endogenous Pdgfra start codon to generate PdgfraDreER allele (Figure 3A). Like the Cre-loxP system, Dre is a heterospecific recombinase that specifically targets rox sites (18, 19). Dre-rox recombination removes rox-flanked transcriptional stop cassette, resulting in permanent expression of tdTomato and irreversible labeling of all descendants $(20,21)$ (Figure 3B). We crossed Pdgfra-DreER to the R26R-rox-tdTomato reporter line (22) and treated adult Pdgfra-DreER R26R-rox-tdTomato mice with tamoxifen to induce PDGFRA ${ }^{+}$cell labeling. Flow cytometric analysis of isolated fibroblasts or endothelial cells showed that approximately $50 \%$ of $\mathrm{PDGFRA}^{+}$fibroblasts without a distinct population of $\mathrm{PECAM}^{+}$endothelial cells were $\mathrm{tdTomato}^{+}$(Figure 3, C and D), suggesting that Pdgfra-DreER efficiently and specifically labeled fibroblasts, but not endothelial cells. After injury, we rarely detected any tdTomato ${ }^{+}$endothelial cells, indicating that PDGFRA ${ }^{+}$fibroblasts did not contribute to endothelial cells in the injured heart (Figure 3D). One reasonable explanation for the presence of the rare tdTomato signal could be autofluorescent background. Whole-mount fluorescence view of hearts showed a significant increase in $\mathrm{tdTomato}^{+}$cells after injury (Figure $3 \mathrm{E}$ ), indicating that injury induced expansion of the PdgfraDreER-labeled cells. Before injury, immunostaining for PECAM or VE-CAD with PDGFRA on heart sections showed that $52.11 \%$ $\pm 2.91 \%$ of PDGFRA $^{+}$fibroblasts were tdTomato $^{+}$and that all $\mathrm{PECAM}^{+}$or VE-CAD ${ }^{+}$endothelial cells were tdTomato ${ }^{-}$(Supplemental Figure 3). After cardiac injury, excessive tdTomato ${ }^{+}$ cells accumulated in the infarct region (Figure 3, E and F), and immunostaining of sections showed that these tdTomato ${ }^{+}$cells still expressed PDGFRA and did not differentiate into PECAM ${ }^{+}$ or $\mathrm{VE}-\mathrm{CAD}^{+}$endothelial cells (Figure 3, G-J). Magnified $Z$-stack confocal image analysis revealed that $\mathrm{td}^{\mathrm{T} o m a t o}{ }^{+}$cells were close to and sometimes attached to endothelial cells, but not exactly colocalized with endothelial cell markers in the heart (Figure 3, $\mathrm{G}^{-} \mathrm{J}$ ). This result was verified in over 500 heart sections from 4 Pdgfra-DreER R26R-rox-tdTomato mice after injury. Microscopic quantification of the labeling percentage of endothelial cells or fibroblasts showed that $0 \%, 0 \%$, and $51.22 \% \pm 5.47 \%$ of $\mathrm{PECAM}^{+}$, $\mathrm{VE}^{-C A D}{ }^{+}$, and PDGFRA ${ }^{+}$cells were tdTomato ${ }^{+}$, respectively $(n=$ 4 , Figure $3 \mathrm{~K}$ ). Our data demonstrated that PDGFRA ${ }^{+}$fibroblasts expanded to generate more fibroblasts, but did not contribute to new blood vessels in the injured heart.

Sox $9^{+}$mesenchymal cells do not contribute to endothelial cells. We next traced the fate of mesenchymal cells using independent tools with an attempt to score for MEndoT during cardiac injury. Previous studies showed that cardiac-resident fibroblasts are largely derivatives of epicardial cells (5) and that the mesenchymal cell marker Sox 9 is an important component of PDGF receptor-mediated signaling during epicardial epithelial-tomesenchymal transition (EMT) (23). We, therefore, generated a Sox9-CreER knockin allele, in which a cDNA encoding Cre and ER fusion protein was inserted into the Exon 3 of Sox 9 gene by internal ribosome entry site (IRES) linking Sox 9 and CreER. We crossed Sox9-CreER with R26R-tdTomato reporter line and test- ed whether this new line could be utilized for tracing mesenchymal cells in MEndoT study. When the Sox9-CreER R26R-tdTomato mice were treated with tamoxifen at the adult stage before injury, Sox9-CreER efficiently labeled the PDGFRA ${ }^{+}$fibroblast population $(88.54 \% \pm 3.77 \%, n=4)$ without labeling endothelial cells marked by PECAM (0 out of 8,216 cells counted, Figure 4, A and B). Before injury, these Sox9-CreER-labeled cells were not $\mathrm{VE}^{-\mathrm{CAD}^{+}}$endothelial cells (Supplemental Figure 4A). We next utilized the Sox9-CreER line to determine whether the labeled Sox $9^{+}$fibroblasts contributed to endothelial cells after cardiac injury. We found a significant expansion of tdTomato ${ }^{+}$cells in the injured region 7 days after cardiac injury (Figure 4C). By immunostaining on injured heart sections, we found that most of the PDGFRA $^{+}$fibroblasts were tdomato $^{+}(82.48 \% \pm 4.24 \%, n=4)$, but negative for PECAM (0 out of 10,378 cells counted, Figure 4D) and VE-CAD (O out of 9,479 cells counted, Supplemental Figure $4 \mathrm{~B}$ ) in the border region of injured hearts. We obtained similar results in both infarct and remote regions of injured hearts (Supplemental Figure 4, C and D). Flow cytometric analysis showed that Sox9-CreER labeled over 85\% PDGFRA ${ }^{+}$fibroblasts after tamoxifen induction (Figure 4E). Similarly, these tdTomato ${ }^{+}$fibroblasts did not contribute to a distinct PECAM $^{+}$ endothelial cell population before and after injury (Figure $4 \mathrm{~F}$ ). Moreover, we detected a significant increase in the percentage of proliferating Sox9+ fibroblasts after injury compared with the uninjured heart $\left(\mathrm{EdU}^{+}{ }^{+} \mathrm{TTomato}^{+}\right.$cell percentage: $0.28 \% \pm 0.12 \%$ vs. $18.85 \% \pm 2.08 \%$; $\mathrm{Ki}^{2} 7^{+} \mathrm{tdTomato}^{+}$cell percentage: $0.33 \% \pm$ $0.12 \%$ vs. $23.51 \% \pm 0.98 \%$, before and after injury, respectively, $n=4$; Figure $4, \mathrm{G}$ and $\mathrm{H}$ ). Taken together, these data suggest that Sox $9^{+}$fibroblasts proliferated to generate more fibroblasts without contribution to endothelial cells in the injured heart.

$\mathrm{TCF} \mathrm{1}^{+}$fibroblasts or POSTN ${ }^{+}$myofibroblasts do not generate new endothelial cells. To further address whether MEndoT happened in vivo, we utilized 2 additional independent tools based on the transcription factor 21 (Tcf21) and periostin (Postn) genes. Recent studies showed that Tcf21-MerCreMer specifically labels fibroblasts in developing and adult hearts $(24,25)$. We performed lineage tracing by generating Tcf21-MerCreMer $R 26 R$ GFP mice in an IR model. Immunostaining for GFP and PECAM showed that there was no $\mathrm{GFP}^{+}$endothelial cell in hearts before and after injury (Supplemental Figure 5A). It has been reported that periostin is highly expressed in the activated cardiac fibroblasts or myofibroblasts of the injured heart, which are derived from tissue-resident fibroblasts of the TCF21 lineage, but not endothelial cells $(6,11)$. We also used Postn-MerCreMer R26RGFP for lineage tracing of myofibroblasts in injured heart and did not detect any $\mathrm{GFP}^{+}$endothelial cells (Supplemental Figure 5B). Flow cytometric analysis of labeled endothelial cells also confirmed that there were rarely any endothelial cells derived from either the $\mathrm{TCF} 21^{+}$or $\mathrm{POSTN}^{+}$cell lineage (Supplemental Figure 5, C and D). Collectively, the above data based on 6 different fibroblast-associated genetic tracing tools indicated that fibroblasts proliferated and expanded after injury, but did not mediate cardiac neovascularization through MEndoT.

Preexisting endothelial cells contribute to neovascularization almost exclusively. It is possible that these Cre lines may not label all cardiac fibroblasts so that a subset of the unlabeled fibro- 
A

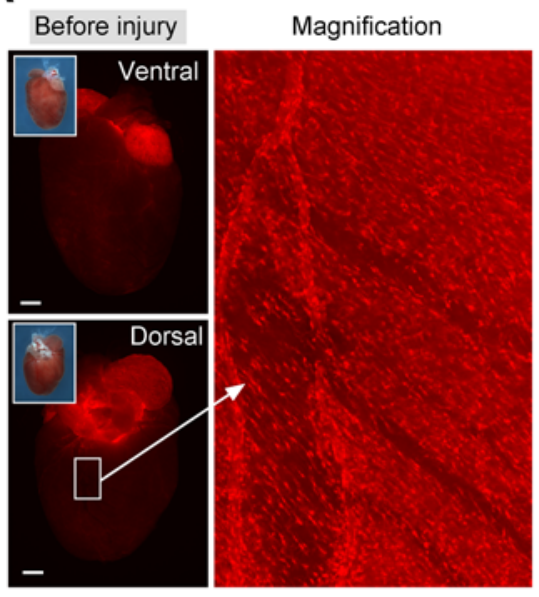

C

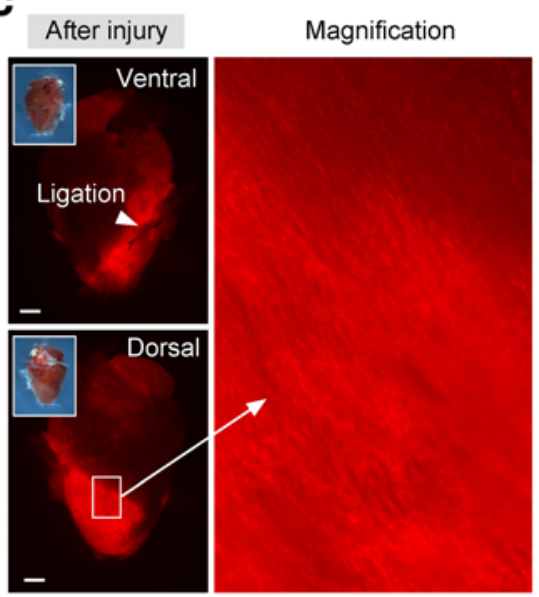

B

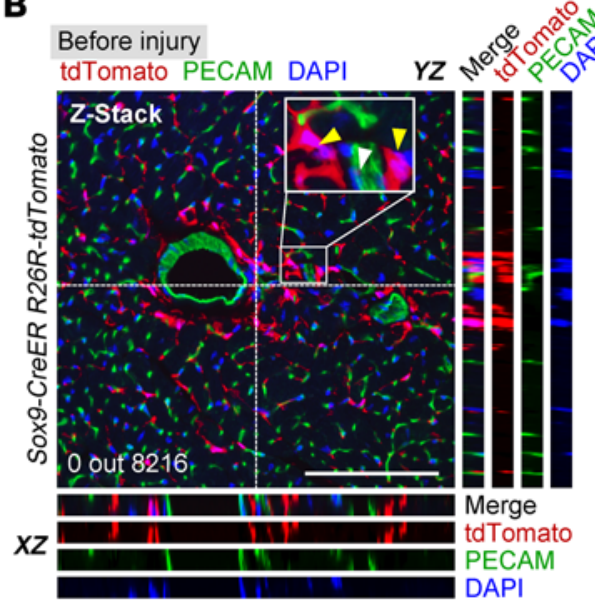

D

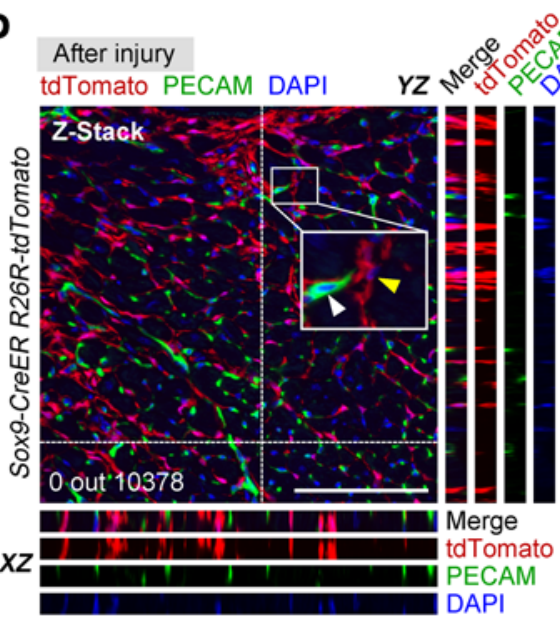

$\mathbf{F}$

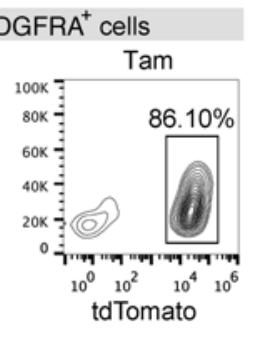

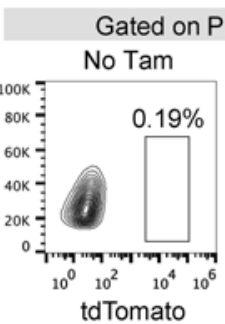

tdTomato

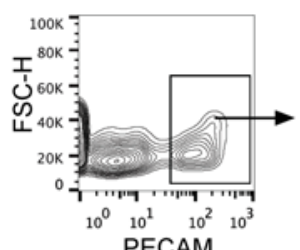

PECAM

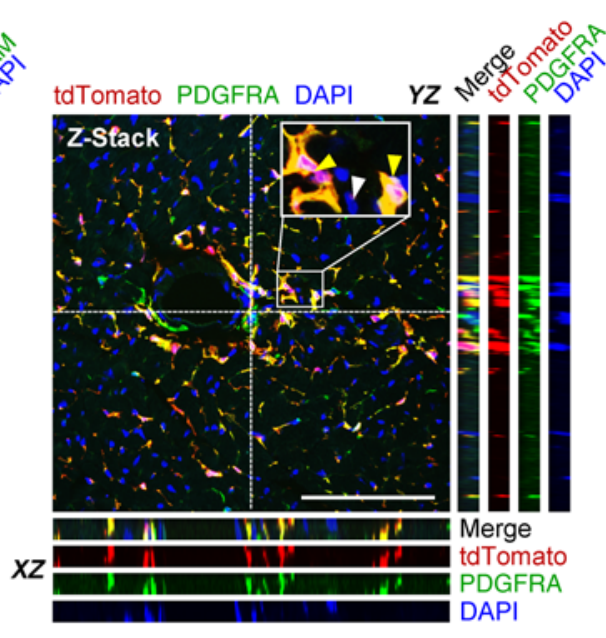

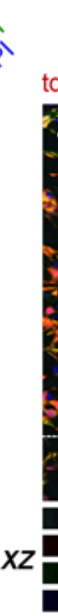
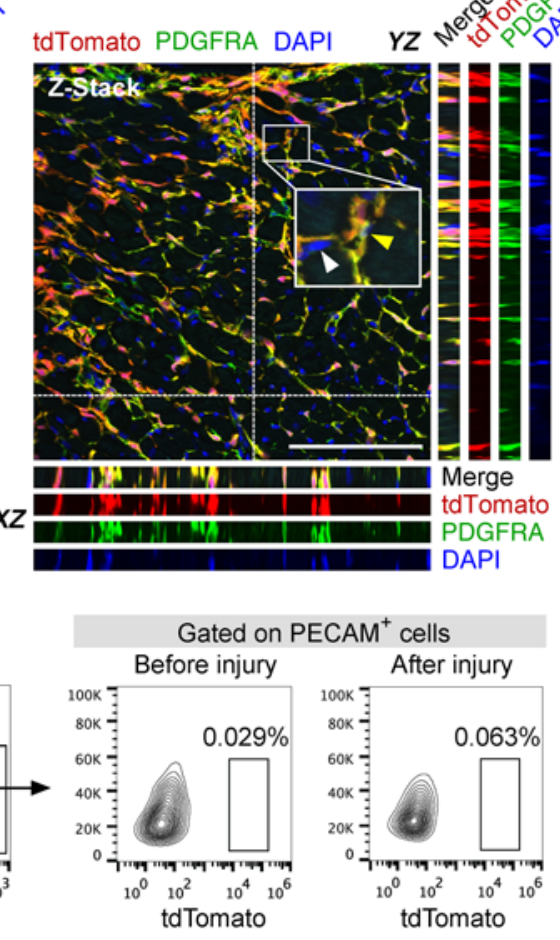

G

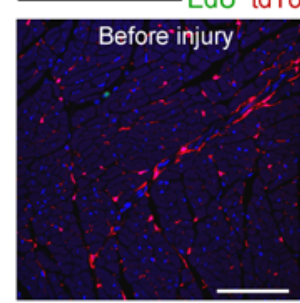

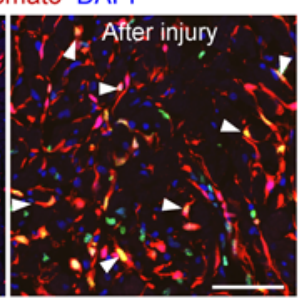

Ki67 tdTomato DAPI

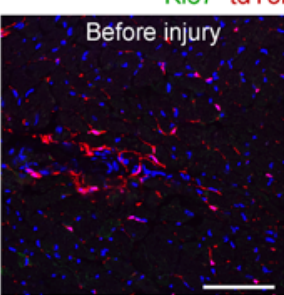

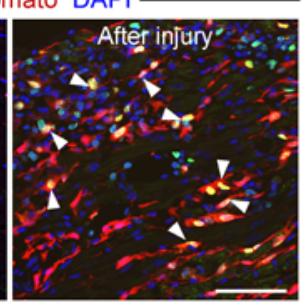

H

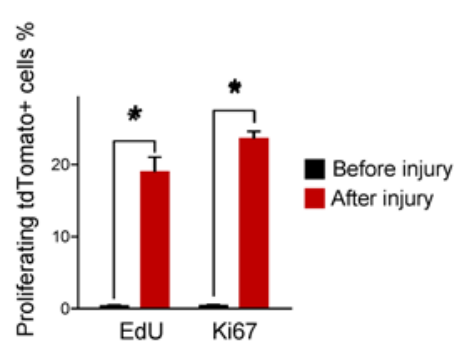

Figure 4. Cardiac fibroblasts expand after injury without giving rise to endothelial cells. (A and C) Whole-mount fluorescence view of Sox9-CreER $R 26 R$-tdTomato hearts before (A) and after injury (C). Inserts indicate bright-field view of hearts. (B and D) Immunostaining for tdTomato, PDGFRA, and PECAM on heart sections before (B) and after injury (D). $X Z$ and $Y Z$ indicate signals from dotted lines on Z-stack images. Yellow arrowheads indicate PDGFRA ${ }^{+}$tdTomato ${ }^{+}$fibroblasts; white arrowheads indicate PECAM+tdTomato endothelial cells. (E) Flow cytometric analysis of percentage of tdTomato cells in PDGFRA ${ }^{+}$cell population. (F) Flow cytometric analysis of tdTomato ${ }^{+}$cells in PECAM ${ }^{+}$endothelial cells from heart before or after injury. (G) Immunostaining for tdTomato and EdU or Ki67 on heart sections before or 3 days after injury. Arrowheads indicate proliferating tdTomato ${ }^{+}$ cells. (H) Quantification of percentage of proliferating tdTomato cells. Data are represented as mean \pm SEM. $n=4$. ${ }^{*} P<0.05$, 2-tailed Student's $t$ test. Scale bars: $1 \mathrm{~mm}$ (A, C); $100 \mu \mathrm{m}$ (B, D, G). 
A

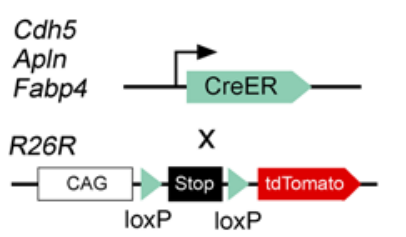

B

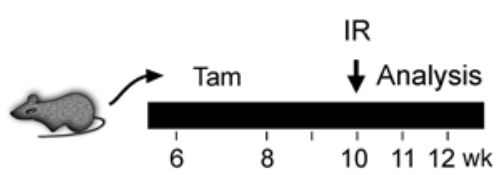

Experimental strategy

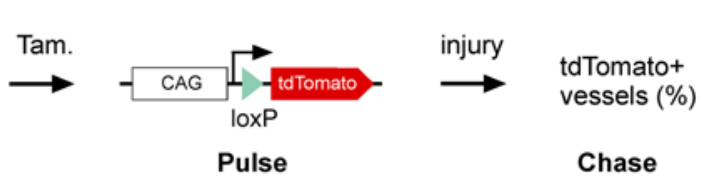

Apln-CreER R26R-tdTomato

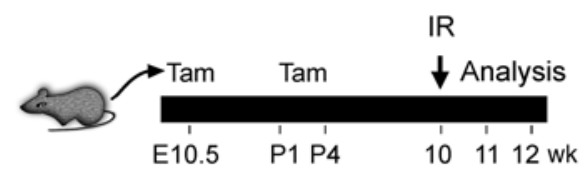

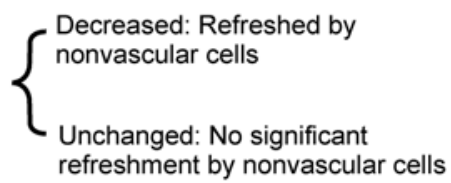

Fabp4-CreER R26R-tdTomato

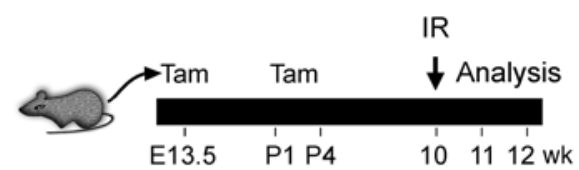

C

tdTomato
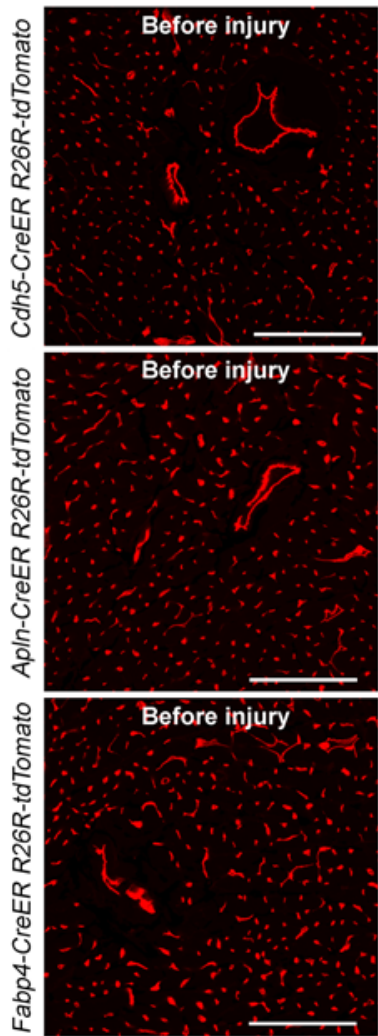

PDGFRA
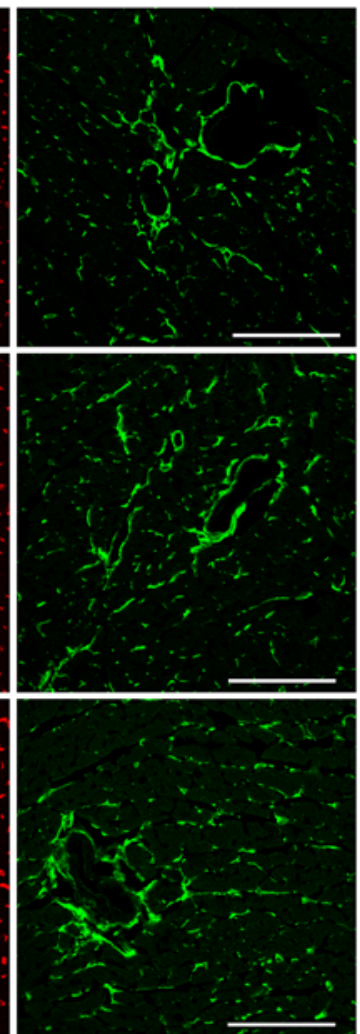

DAPI
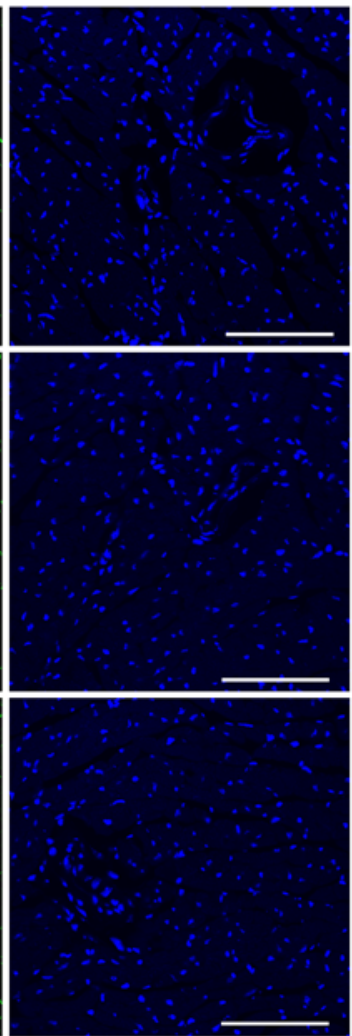

tdTomato PDGFRA DAPI
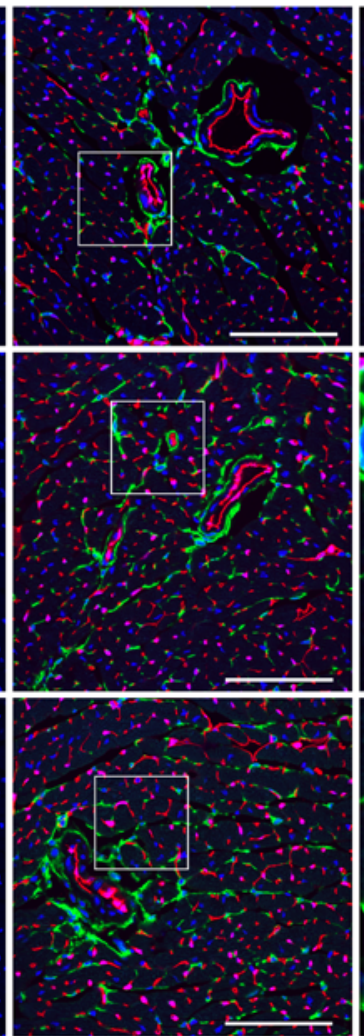

Magnification
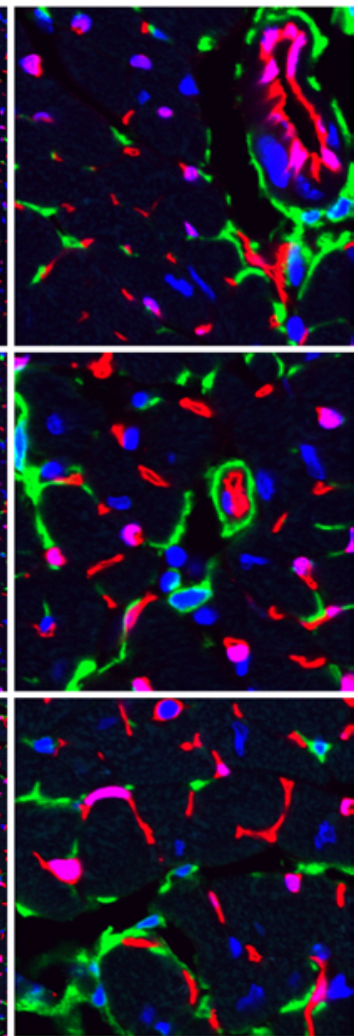

Figure 5. Establishment of pulse-chase strategy for labeling of coronary endothelial cells. (A) Schematic figure showing the pulse-chase experimental strategy for measuring refreshment of nonvascular cells. (B) Experimental design for tamoxifen induction, myocardial IR, and tissue analysis. wk, postnatal weeks. (C) Immunostaining for tdTomato and PDCFRA on tissue sections shows that these Cre lines do not label fibroblasts before injury. Each image is representative of 4 individual samples. Scale bars: $100 \mu \mathrm{m}$.

blasts may undergo MEndoT to generate new blood vessels after injury. If MEndoT did contribute significantly to neovascularization of the injured heart, as previously reported in a study where approximately $30 \%-40 \%$ of fibroblasts adopted the endothelial cell fate (9), there should be a substantial number of fibroblastderived endothelial cells infiltrated into the preexisting coronary vasculature. To independently address this issue, we designed a series of new experiments via "pulse-chase" genetic fate-mapping (26). Expression of the inducible Cre in endothelial cells enabled us to genetically and specifically label preexisting vessels, but not cardiac fibroblasts, before injury. If a substantial number of cardiac fibroblasts contributed to blood vessels after injury (9), there should be a reasonable number of unlabeled new coronary vessels derived from these unlabeled fibroblasts, resulting in dilution of the vascular labeling efficiency in the injured hearts (Figure 5A). If the labeling efficiency in coronary vessels remained relatively constant after injury, we could interpret that to mean that cardiac fibroblasts did not contribute significantly to coronary vessels after injury (Figure $5 \mathrm{~A}$ ). Three different lineage-tracing models for coronary endothelial cells via inducible Cre were therefore examined in this study: Cdh5CreER (27), Apln-CreER (where Apln indicates apelin) (28), and 

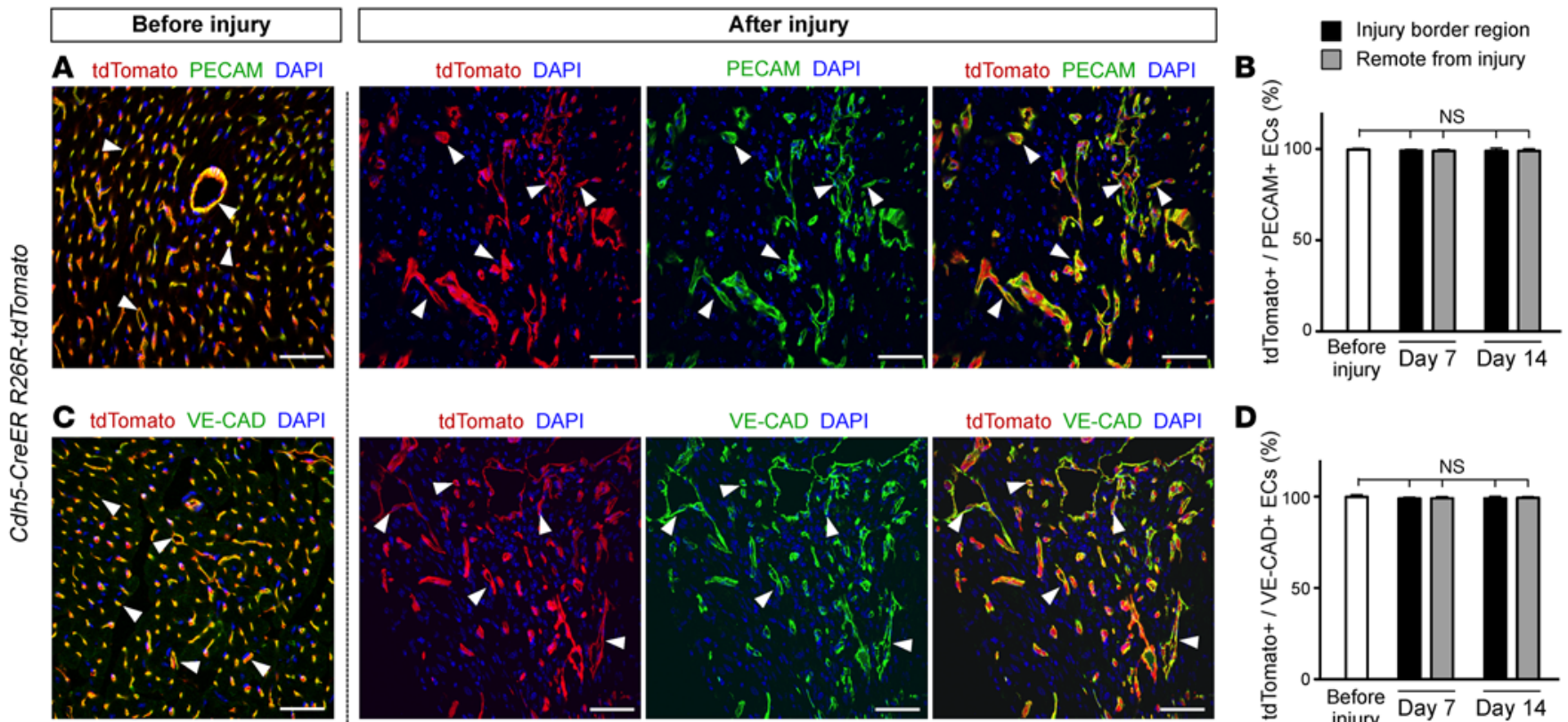

tdTomato VE-CAD DAPI
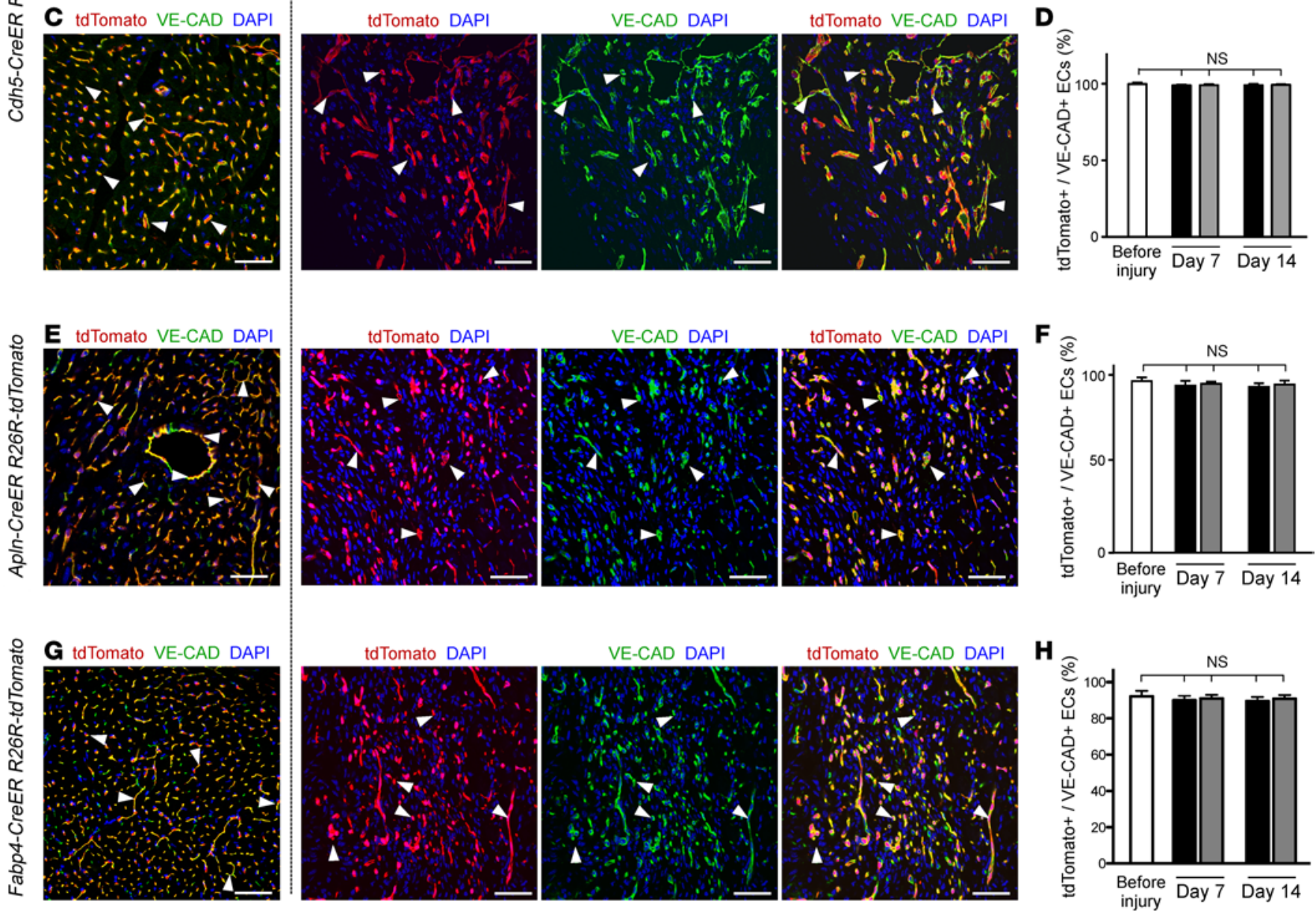

Figure 6. Coronary vessels in the injured heart are derived from preexisting coronary vessels. (A, C, E, C) Immunostaining for tdTomato and PECAM or VE-CAD on heart sections before and after injury. Arrowheads indicate labeled endothelial cells (tdTomato ${ }^{+} P E C A M^{+}$or tdTomato $\left.{ }^{+} V E-C A D+\right)$. (B, D, F, H) Quantification of the percentage of tdTomato endothelial cells (ECs) in PECAM+ or VE-CAD+ endothelial cells in hearts before injury and 7 or 14 days after injury. Data are represented as mean \pm SEM. $n=4$. ANOVA test was used. Scale bars: $50 \mu \mathrm{m}$.

Fabp4-CreER (where Fabp4 indicates fatty acid-binding protein 4) (29). Cdh5 (encoding VE-CAD) is a specific endothelial cell marker (27). Apln is a specific marker for vascular endothelial cells $(30,31)$, and Apln-CreER has been utilized to mark vascular endothelial cells $(32,33)$. Fabp 4 is expressed in adipocytes of fat tissue and is also highly enriched in vascular endothelial cells (34-36). To further prove the vascular endothelial specificity of FABP4, we performed immunostaining for FABP4 with VE-CAD or PDGFRA. Immunostaining data demonstrated that FABP4 was specifically expressed in VE-CAD ${ }^{+}$endothelial cells, but not PDGFRA $^{+}$fibroblasts of the heart (Supplemental Figure 6).
We next used these 3 endothelial inducible Cre lines to trace coronary endothelial cells in the injured heart. To trace them with a high efficiency, we tried different tamoxifen induction strategies that ranged from embryonic and neonatal to adult stages. We finally adopted 3 optimized tamoxifen induction strategies for Cdh5-CreER, Apln-CreER, and Fabp4-CreER (Figure 5B) that could achieve high efficiency of labeling. At week 10, all mice were subjected to an IR injury model, and heart samples were collected at 1 or 2 weeks after injury for analysis (Figure 5B). One important condition for interpretation of the "pulse-chase" experiments is that the labeling of coronary endothelial cells is specific without 


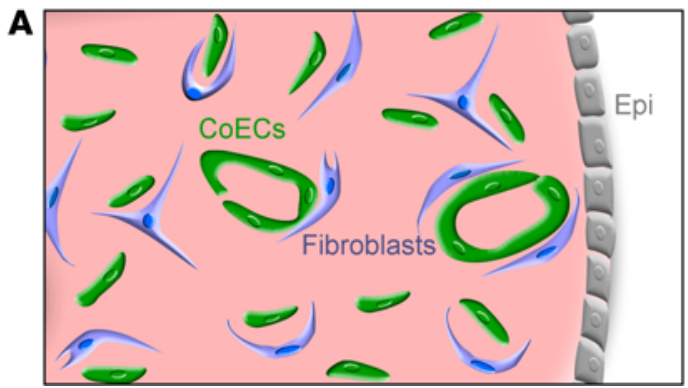

B

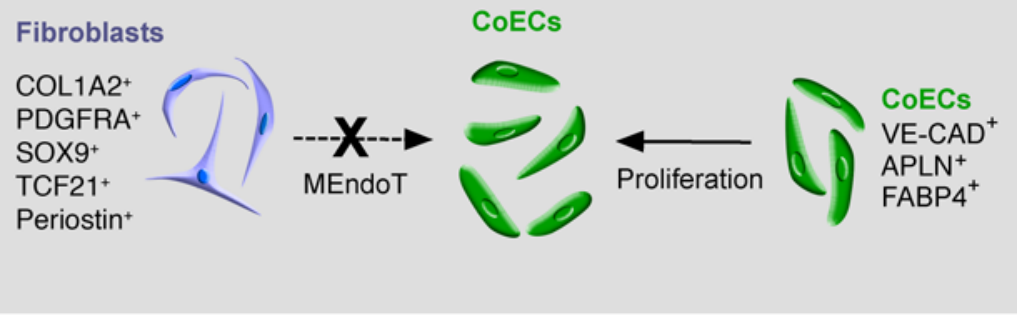

Figure 7. Preexisting coronary endothelial cells but not fibroblasts contribute to blood vessels after injury. (A) Image showing coronary endothelial cells (CoECs) and fibroblasts in the adult heart. Epi, epicardium. (B) Image showing contribution of different lineages to coronary endothelial cells after injury.

"ectopic" labeling of any fibroblasts at baseline. We therefore performed immunostaining for tdTomato and PDGFRA on heart sections before injury and found that these tdTomato cells were not PDGFRA ${ }^{+}$in over 500 heart sections from 4 Cdh5-CreER R26R-tdTomato, 4 Apln-CreER R26R-tdTomato, and 4 Fabp4CreER R26R-tdTomato mice, respectively (Figure 5C), which is in contrast to the fibroblast-specific lineage-tracing models (Colla2CreER, Col1a2-2A-CreER, Pdgfra-DreER, Sox9-CreER, Tcf21-MerCreMer, or Postn-MerCreMer).

We next performed immunostaining for tdTomato with PECAM or VE-CAD and found that almost all coronary endothelial cells were tdTomato ${ }^{+}$in Cdh5-CreER R26R-tdTomato, Apln-CreER R26R-tdTomato, or Fabp4-CreER R26R-tdTomato heart samples, demonstrating that these CreER lines specifically and efficiently labeled coronary vascular endothelial cells before injury (Figure 6, A, C, E, and G). We next examined tissue samples from injured hearts. Using all these inducible Cre lines, we did not detect any significant dilution of the $\mathrm{PECAM}^{+}$or $\mathrm{VE}-\mathrm{CAD}^{+}$coronary vessel labeling in the injury border zone at day 7 or 14 after injury ( $n=4$, Figure $6, \mathrm{~A}-\mathrm{H})$, suggesting that nonendothelial cell lineages including fibroblasts did not contribute significantly to new coronary vessels after injury. Neither did we observe significant labeling in the sham-treated or injured hearts without tamoxifen treatment (Supplemental Figure 7A). Moreover, we obtained similar results via immunostaining for additional endothelial cell markers BS1 lectin and VEGFR2 (Supplemental Figure 7, B-D). In the remote region of the injured heart, we did not find any significant dilution of endothelial cell labeling (Supplemental Figure 8). In the injured heart, we detected a significant increase in $\mathrm{Ki}^{+} \mathrm{t}^{+} \mathrm{dTomato} \mathrm{O}^{+}$or EdU ${ }^{+} \mathrm{td} \mathrm{Tomato}^{+}$endothelial cells (Supplemental Figure 9), indicating expansion of coronary endothelial cellmediated cardiac neovascularization after injury. Taken together, these data demonstrate that most coronary vessels in the injured hearts were derived from the preexisting coronary vessels, but not from the nonendothelial cells through cell transdifferentiation, such as fibroblasts via MEndoT process.

\section{Discussion}

To date, 2 different models have been proposed to explain the formation of new vasculature in a damaged heart, namely expansion of preexisting vessels and cell transdifferentiation from other lineages, such as fibroblasts through MEndoT. Elucidating the sources for neovascularization after cardiac injury would provide impor- tant insights into therapeutic deployment for cardiac regeneration. In this study, we observed that almost no coronary vessels arose from cardiac fibroblasts, and we did not observe any significant conversion of nonendothelial cells into endothelial cells after injury (Figure 7). Resident fibroblasts mainly mediated cardiac fibrosis after injury without contributing to new coronary endothelial cells. Only preexisting coronary endothelial cells begot new coronary vessels in the adult mouse heart, with essentially no contribution from other cell sources through cell-lineage transdifferentiation.

The evidence supporting MEndoT is largely based on tracing experiments by Col1a2-CreER and fibroblast-specific 1 (FSP1) transgene (9). This Col1a2-CreER transgene is generated by placing cDNA encoding CreER under the promoter of Col1a2 and is reported to target resident fibroblasts specifically (14). We also obtained the same line used for MEndoT as previously reported (9) and found that fibroblasts labeled by the Col1a2-CreER transgene did not contribute to any endothelial cells. The exact reason of failing to recapitulate MEndoT using the same line in our study remains unclear. We reason that direct comparison could be unfair because the transgene might have been silenced or the copy number might have been changed when the same mouse line was bred in different laboratories. We then generated a Col1a2-2A-CreER knockin line in this study to reassess MEndoT after cardiac injury. Consistent with the previous report (9), Col1a2-2A-CreER labeled fibroblasts but not endothelial cells before injury. However, we did not detect any labeled coronary endothelial cells after injury and therefore questioned whether MEndo T was a presumptive mechanism for neovascularization after injury. The second tool used to support MEndoT is FSP1-mediated lineage tracing (9). The use of Fsp1-Cre for fibroblast labeling is problematic, as FSP1 is also expressed by endothelial cells in situ in addition to fibroblasts, consistent with the previous study (37). Moreover, since Fsp1-Cre contains the constitutive Cre, any endothelial cells reactivating FSP1 expression after injury would also be genetically labeled. Therefore, increased labeling of endothelial cells by Fsp1-Cre lineage tracing could not solely demonstrate fibroblast-to-endothelial cell transition. These data need to be interpreted with caution, given caveats of genetic-lineage tracing $(38,39)$.

Our genetic-tracing study of coronary endothelial cells showed that they proliferated and expanded substantially after injury and that there was not any significant dilution of vascular labeling after injury. These data demonstrated that virtually all coronary endothelial cells after injury were derived from preexisting coronary 
endothelial cells and that nonendothelial cell lineage minimally contributed to coronary endothelial cells. One caveat for interpretation of these dilution experiments would be that a small number of nonendothelial cells could have differentiated into endothelial cells $(<1 \%)$ and our experimental strategy was not sensitive enough to reveal its contribution. This possibility could not be excluded by our current lineage-tracing system. Nevertheless, since we have labeled most endothelial cells and these labeled cells still constituted a similar percentage of endothelial cells after injury, we interpret that endothelial cells contributed to most, if not all, neovascularization in the injured heart. One may ask whether cardiac stem cells such as $\mathrm{Kit}^{+}$cells, Sca1 ${ }^{+}$cells, or epicardial progenitors contribute to new coronary endothelial cells after cardiac injury. As for $\mathrm{Kit}^{+}$cells, genetic-lineage-tracing studies showed that Kit-Cre labels a substantial number of endothelial cells in the normal heart and that those cells expand to generate more coronary endothelial cells after injury (40-42). Whether $\mathrm{Kit}^{+}$nonendothelial cells could give rise to new vessels remains to be detected by new genetic tools that could distinguish $\mathrm{Kit}^{+}$nonendothelial cells from $\mathrm{Kit}^{+}$endothelial cells before injury. Nevertheless, our dilution study suggested that even if $\mathrm{Kit}^{+}$nonendothelial cells generate new endothelial cells, the number is restricted so that it is minimal. Previous studies suggest that $\mathrm{Sca}^{+}$cells are regarded as cardiac progenitor cells that form multiple lineages in adult heart (43), and lineage-tracing studies show that Sca1-derived cells are most endothelial cells in the normal heart (44). Our endothelial cell-tracing study suggests that these $\mathrm{Sca}^{+}$endothelial cells might expand after injury and that Sca1 ${ }^{+}$nonendothelial cells minimally contributed to coronary endothelial cells. It is also possible that, during injury, mature endothelial cells respond to ischemic microenvironment and reexpress progenitor markers such as c-Kit and Sca1. Adult epicardial progenitors were reported to contribute significantly to fibroblasts after injury and rarely to coronary endothelial cells (45), which is consistent with our endothelial tracing study.

In this study, we utilized 6 different genetic tracing tools to label fibroblasts, but were unable to recapitulate the role of MEndoT in vascular regeneration. Additional data based on 3 independent endothelial cell-specific inducible Cre lines supported the conclusion that preexisting coronary endothelial cells were the major source for cardiac neovascularization after injury (Figure 7). Our work suggests that caution should be used in attempting to develop clinical applications solely based on the cell-lineage conversion paradigm for treatment of cardiac diseases. On the other hand, our data suggested that preexisting vessels appeared more likely to be the therapeutic target for promoting neovascularization and driving heart regeneration after injury.

\section{Methods}

Mice. Col1a2-CreER transgene (14), Tcf21-MerCreMer (24), PostnMerCreMer (6), Cdh5-CreER (27), Apln-CreER (28), Fabp4-CreER (29), R26R-tdTomato (16), R26R-rox-tdTomato (22), and R26R-GFP (46) mouse lines were previously described. Sox 9 -CreER was generated by homologous recombination using CRISPR/Cas9 methods (21). A cDNA encoding IRES-CreERT2-polyA cassette was introduced into the Hpa1 site within the 3'-UTR of Sox9 exon3, as previously described (47). For Colla2-2A-CreER allele, a cDNA encoding $\mathrm{CreER}^{\mathrm{T} 2}$ recombinase was inserted into the translational stop codon of the Col1a2 gene, following endogenous Col1a2 by the self-cleaving peptide 2A sequence. For the Pdgfra-DreER allele, a cDNA encoding DreER $^{\mathrm{T} 2}$ recombinase was inserted after the translational stop codon of the Pdgfra gene, as previously described (48). All mice were maintained on an ICR/C57BL6/J-mixed background. Tamoxifen (1 mg) (Sigma-Aldrich) was injected intraperitoneally for 10 days to induce Cre-mediated recombination in Col1a2-CreER R26R-tdTomato mice as previously described (9). For other inducible Cre lines, tamoxifen (Sigma-Aldrich) was dissolved in corn oil $(20 \mathrm{mg} / \mathrm{ml})$ and administered by oral gavage before injury or in sham operations $(0.1-0.15$ mg tamoxifen/g mouse body weight). Specifically, Col1a2-2A-CreER R26R-tdTomato, Pdgfra-DreER R26R-rox-tdTomato, Sox9-CreER R26R-tdTomato, and Cdh5-CreER R26R-tdTomato were treated with tamoxifen 5 times within 10 days at 6 to 8 weeks old. Two weeks following cessation of tamoxifen, these mice were subjected to IR cardiac injury (described below). For Tcf21-MerCreMer and Postn-MerCreMer, Cre recombinase activity was induced with tamoxifen citrate-containing chow at a treatment dosage of $400 \mathrm{mg} / \mathrm{kg}^{-1}$ (Envigo, TD.130860). Tcf21-MerCreMer R26R-GFP mice received TAM food for 2 weeks prior to injury and then stopped at time of injury until the mice were harvested 2 weeks later. Sham-treated mice were also given tamoxifen for 2 weeks, then given a sham surgical procedure, and again, hearts were removed 2 weeks later. Postn-MerCreMer R26R-GFP mice received TAM food subsequent to the IR surgery continuously for the following 2 weeks of reperfusion until harvest. After 2 weeks of reperfusion, mice were sacrificed by $\mathrm{CO}_{2}$ asphyxiation and the harvested hearts were washed in sterile PBS and either fixed and processed for histology or digested and analyzed with flow cytometry as described below. For Apln-CreER R26R-tdTomato mice, tamoxifen was administered at E10.5, P1, and P4 to increase its labeling efficiency, and mice were subjected to IR cardiac injury at 10 weeks old. For Fabp4-CreER R26RtdTomato mice, tamoxifen was administered at E13.5, P1, and P4 to increase its labeling efficiency, and mice were subjected to IR cardiac injury at 10 weeks old. In this study, both male and female mice were included in all experiments.

Cardiac injury model. Myocardial IR cardiac injury was performed in adult mice as described previously (9). Mice (both male and female), 8 to 10 weeks old, were randomly allocated to the sham or IR cardiac injury group. Investigators performing surgeries and sample analysis were blinded to mouse genotype and treatment. Anesthesia was induced by tracheal intubation and administration of $2 \%$ isoflurane gas, while the core temperature of the animal was maintained by placement upon a $37^{\circ} \mathrm{C}$ water-heated pad. The ventilator respiratory rate was set between 120 and 130, and the respiratory volume to $0.3-0.5$ $\mathrm{ml}$. Upon reaching a surgical plane of anesthesia, the isoflurane concentration was adjusted to $1.5 \%$. We confirmed the mice were ready for surgery by pain reflex reaction (lack of response to hind limb toe pinch). The limbs were then fixed in place, and chest skin was disinfected. A vertical 1- to $1.5-\mathrm{cm}$ incision was made at the sternum, and then a blunt dissection of the muscle and fascia was performed to avoid injuring the blood vessels. After making an approximately $1-\mathrm{cm}$ incision between the third and fourth intercostal ribs, the chest was expanded to approximately $0.8-\mathrm{cm}$ wide. Then the left anterior descending (LAD) branch of the coronary artery was ligated with an 8-0 suture, and a 0.1-cm hose was inserted in between the suture and the LAD ligated site during ligation. The ligation was judged as successful when the heart showed signs of cyanosis. After 30 minutes, the LAD coronary artery was reper- 
fused by removing the hose and suture. Then the ribs and skin incisions were closed with a 6-0 suture and any bubbles were discharged from the chest. The surgical incision was disinfected and mice were supplied with pure oxygen for 4 to 5 minutes until they resumed spontaneous breathing. Then the tracheal intubation tube was removed and the mice were kept warm until recovery of normal behavior was observed, followed by analgesic treatment. Sham injury was performed in the same manner, with a ligature passed under the LAD, but the LAD was not ligated. At 7 or 14 days after injury, the mouse hearts were harvested for analysis. No animals were excluded from analysis unless they died during or after the surgical procedure. Cardiac infarction was verified by sectional Sirius red staining assays.

Immunostaining and Z-stack confocal microscopy. Immunostaining was performed as previously described (32). Briefly, hearts were collected in PBS on ice and then fixed in $4 \%$ paraformaldehyde at $4^{\circ} \mathrm{C}$ for 1 hour. After washing in PBS, tissues were incubated in PBS $/ 30 \%$ sucrose overnight at $4^{\circ} \mathrm{C}$ and embedded in optimum cutting tissue (OCT) (Sakura) for snap-frozen procedure the following day. Cryosections of $10-\mu \mathrm{m}$ thickness were collected on positively charged slides. Tissues were blocked with PBS/0.1\% Triton X-100/5\% normal donkey serum (Jackson ImmunoResearch) for 1 hour at room temperature, followed by primary antibody incubation overnight at $4^{\circ} \mathrm{C}$. Signals were visualized with Alexa Fluor-conjugated secondary antibodies (Invitrogen). For weak signals, we used HRP- or biotinconjugated secondary antibodies and a tyramide signal amplification kit (PerkinElmer). Antibodies used were as follows: PECAM/ CD31 (BD Biosciences - Pharmingen, 553370), tdTomato (Rockland, 600-401-379), GFP (Invitrogen, A11122), FABP4 (Abcam, ab28723), VE-CAD (R\&D, AF1002), PDGFRA (R\&D, AF1062; eBioscience, 14-1401-81), DDR2 (Santa Cruz Biotechnology Inc., sc-7555), Ki67 (Thermo Scientific, RM-9106), and VEGFR2 (BD Biosciences - Pharmingen). For BS1 lectin perfusion, $1.25 \mu \mathrm{l}$ BS1 lectin (Vector Laboratories) per gram of body weight dissolved in 200 $\mu \mathrm{PBS}$ was injected in the inferior vena cava and hearts were harvested 30 minutes later. We used the Click-It EdU Imaging Kit (Life Technology) for detection of EdU incorporation according to the manufacturer's instructions. EdU was injected intraperitoneally into the mice 24 hours before sacrifice at a dose of $10 \mu \mathrm{g} / \mathrm{g}$ mouse body weight. Images were acquired by Olympus confocal microscope (FV1000), Zeiss confocal microscope (LSM510), Leica confocal microscope (TCS SP5), or a Zeiss stereo microscope (AXIO Zoom, V16). Z-stack images were obtained as previously described (49). Briefly, to provide $Z$-stack confocal images, we scanned 5-7 consecutive $X Y$ images on the $Z$ axis with an Olympus confocal microscope FV1000. The obtained image data were analyzed by Image (NIH) software. The images were merged using the Image color-merge channels function, and then stacks were done using $Z$-projects and average intensity projection. In the stack, the orthogonal view was adjusted to reveal the signals on the $X Z$ and $Y Z$ axes. Merged signals and split channels were shown to delineate the signals on single-cell resolution. All quantification work was performed by an observer blinded to the experimental designs.

Noncardiomyocyte isolation. Adult noncardiomyocytes were isolated as described previously (40). Briefly, adult mice were injected with $200 \mu \mathrm{l}$ heparin $(6.25 \mathrm{U} / \mu \mathrm{l})$ intraperitoneally to prevent the coronary arteries from being blocked. Fifteen minutes later, the mice were anesthetized by intraperitoneal injection of pentobarbital sodium at
$80 \mathrm{mg} / \mathrm{kg}$ body weight. After 5 to 10 minutes, the hearts were dissected and ligated to the perfusion fluid-filled aortic cannula. The hearts were perfused with perfusion buffer $(137 \mathrm{mM} \mathrm{NaCl}, 4 \mathrm{mM} \mathrm{KCl}$, $0.33 \mathrm{mM} \mathrm{NaH}_{2} \mathrm{PO}_{4}, 1 \mathrm{mM} \mathrm{MgCl}, 10 \mathrm{mM}$ HEPES, $5 \mathrm{mM}$ taurine, 10 $\mathrm{mM} \mathrm{BDM}$, and $10 \mathrm{mM}$ glucose, $\mathrm{pH}$ 7.4) at a flow rate of $4 \mathrm{ml} / \mathrm{min}$ for about 5 minutes until the effluents became clear. Then the hearts were perfused with digestion buffer (perfusion buffer containing $0.2 \mathrm{mg} /$ $\mu \mathrm{l}$ collagenase II and $0.16 \mathrm{mg} / \mu \mathrm{l}$ Protease XIV) for 15 to 20 minutes at the same flow rate. When the hearts became pale and swelling, the digested hearts were transferred to transfer buffer (perfusion buffer containing $0.5 \mathrm{mg} / \mathrm{ml} \mathrm{BSA}$ ) and minced by forceps and filtered through a $70-\mu \mathrm{m}$ strainer. The isolated cells were centrifuged for 3 minutes at $20 \mathrm{~g}$ to separate out the pellets that contained cardiomyocytes, and then the supernatant containing noncardiomyocytes was centrifuged as pellets at $500 \mathrm{~g}$ for 10 minutes at $4^{\circ} \mathrm{C}$; the pellets were subsequently resuspended in transfer buffer for further analysis.

Flow cytometry. Flow cytometric analysis was performed as previously described (50). Isolated noncardiomyocytes were treated with red blood cell lysis buffer (eBioscience) for 5-10 minutes at room temperature to lyse red blood cells. After that, remaining cells were incubated with anti-mouse CD16/CD32 to block endogenous Fc for 5 minutes at room temperature. After this, cells were stained with antibodies including CD45-FITC (11-0451, eBioscience), PDGFRA-APC (17-1401, eBioscience), PECAM-PE-Cy7 (25-0311, eBioscience), or PECAM-APC (17-0311, eBioscience) for 30 minutes at $4^{\circ} \mathrm{C}$. Then the cells were treated with DAPI to stain the dead cells. Finally, the cells were resuspended with $300 \mu$ isolation buffer. We analyzed stained cells on a MoFlo Astrios Flow Cytometer System (Beckman Coulter), BD LSR II, or BD FACSCanto II Flow Cytometer System, and obtained data were analyzed by FlowJo software (TreeStar).

Statistics. All data were determined from 4 independent samples and presented as mean \pm SEM. Statistical comparisons between data sets were made with analysis of normality and variance, followed by a 2-sided unpaired Student's $t$ test for comparing differences between 2 groups and ANOVA test for over 2 groups. $P<0.05$ was considered significant. All mice were randomly assigned to different experimental groups.

Study approval. This study was carried out in strict accordance with the recommendations set forth in the Guide for the Care and Use of Laboratory Animals of the CAS. The protocol was approved by the IACUC of the Institute for Nutritional Sciences and the Institute of Biochemistry and Cell Biology, Shanghai Institutes for Biological Sciences, CAS. All efforts were made to minimize animal suffering.

\section{Author contributions}

$\mathrm{LH}$ and BZ designed the study, performed experiments, and analyzed the data. XH, OK, YL, YW, YL, WP, QL, H Zhang, XT, H Zhao, XL, and SZ bred the mice and performed experiments. YN, $\mathrm{SH}, \mathrm{XM}$, and QDW analyzed the data, provided technical support, and reviewed and edited the manuscript, FW and TC provided a mouse line. QX, KOL, and JDM analyzed the data, provided intellectual input, and edited the manuscript, BZ conceived and supervised the study, analyzed the data, and wrote the manuscript.

\section{Acknowledgments}

We thank Ralf Adams and Hongkui Zeng for sharing the mouse lines. We thank Shanghai Biomodel Organism Co. Ltd. for mouse generation. We express our appreciation to Baojin $\mathrm{Wu}$, Guoyuan 
Chen, Zhonghui Weng, and Aimin Huang for animal husbandry; Wei Bian for technical help; and Juan Tang for critical reading. We also thank other members of our laboratory for insightful discussion and technical help throughout this study. This work was supported by the Strategic Priority Research Program of the Chinese Academy of Sciences (CAS) (XDB19000000), the National Science Foundation of China (91639302, 31625019, 91339104, 31271552, 31222038, 31571503, 31501172, 31601168, and 81300340), the Ministry of Science and Technology of China (2013CB945302, 2016YFC1300600, and SQ2017YFSF080041), the Youth Innovation Promotion Association of CAS (2015218), the Key Project of Frontier Sciences of CAS (QYZDB-SSWSMC003), the International Cooperation Fund of CAS, the Shang- hai Zhangjiang Stem Cell Research Project (ZJ2014-ZD-002), the Shanghai Science and Technology Commission (14JC1407400, 16DZ2280800, 17ZR1449600, and 17ZR1449800), the Shanghai Yangfan Project (15YF1414000) and Rising-Star Program (15QA1404300), the China Postdoctoral Science Foundation (2015M581669, 2016T90387, and 2016LH0042), the President Fund of Shanghai Institutes for Biological Sciences (SIBS), Astrazeneca, Boehringer Ingelheim, and a Sanofi-SIBS fellowship.

Address correspondence to: Bin Zhou, Institute of Biochemistry and Cell Biology, Chinese Academy of Sciences, 320 Yueyang Road, Life Science Research Building A-2112, Shanghai, China, 200031. Phone: 86.21.54920974; Email: zhoubin@sibs.ac.cn.
1. Hansson GK. Inflammation, atherosclerosis, and coronary artery disease. $N$ Engl J Med. 2005;352(16):1685-1695.

2. Lin YD, et al. Instructive nanofiber scaffolds with VEGF create a microenvironment for arteriogenesis and cardiac repair. Sci Transl Med. 2012;4(146):146ra109.

3. Riley P. Developmental biology: Plumbing the heart. Nature. 2010;464(7288):498-499.

4. Kurian L, et al. Conversion of human fibroblasts to angioblast-like progenitor cells. Nat Methods. 2013;10(1):77-83.

5. Moore-Morris T, et al. Resident fibroblast lineages mediate pressure overload-induced cardiac fibrosis. J Clin Invest. 2014;124(7):2921-2934.

6. Kanisicak O, et al. Genetic lineage tracing defines myofibroblast origin and function in the injured heart. Nat Commun. 2016;7:12260.

7. Margariti A, et al. Direct reprogramming of fibroblasts into endothelial cells capable of angiogenesis and reendothelialization in tissueengineered vessels. Proc Natl Acad Sci U S A. 2012;109(34):13793-13798.

8. Han JK, et al. Direct conversion of adult skin fibroblasts to endothelial cells by defined factors. Circulation. 2014;130(14):1168-1178.

9. Ubil E, et al. Mesenchymal-endothelial transition contributes to cardiac neovascularization. Nature. 2014;514(7524):585-590.

10. Miyake T, Kalluri R. Cardiac biology: Cell plasticity helps hearts to repair. Nature. 2014;514(7524):575-576

11. Kaur H, et al. Targeted ablation of periostinexpressing activated fibroblasts prevents adverse cardiac remodeling in mice. Circ Res. 2016;118(12):1906-1917.

12. Ali SR, et al. Developmental heterogeneity of cardiac fibroblasts does not predict pathological proliferation and activation. Circ Res. 2014;115(7):625-635.

13. Pinto AR, et al. Revisiting Cardiac Cellular Composition. Circ Res. 2016;118(3):400-409.

14. Zheng B, Zhang Z, Black CM, de Crombrugghe B, Denton CP. Ligand-dependent genetic recombination in fibroblasts: a potentially powerful technique for investigating gene function in fibrosis. Am J Pathol. 2002;160(5):1609-1617.

15. Duan J, et al. Wnt1/ $\beta$ catenin injury response activates the epicardium and cardiac fibroblasts to promote cardiac repair. EMBO J. 2012;31(2):429-442.
16. Madisen L, et al. A robust and high-throughput Cre reporting and characterization system for the whole mouse brain. Nat Neurosci. 2010;13(1):133-140.

17. Guo C, Yang W, Lobe CG. A Cre recombinase transgene with mosaic, widespread tamoxifeninducible action. Genesis. 2002;32(1):8-18.

18. Anastassiadis K, et al. Dre recombinase, like Cre, is a highly efficient site-specific recombinase in E. coli, mammalian cells and mice. Dis Model Mech. 2009;2(9-10):508-515.

19. Madisen L, et al. Transgenic mice for intersectional targeting of neural sensors and effectors with high specificity and performance. Neuron. 2015;85(5):942-958.

20. Sauer B, McDermott J. DNA recombination with a heterospecific Cre homolog identified from comparison of the pac-c1 regions of P1-related phages. Nucleic Acids Res. 2004;32(20):6086-6095.

21. Zhang $\mathrm{H}$, et al. Genetic lineage tracing identifies endocardial origin of liver vasculature. Nat Genet. 2016;48(5):537-543.

22. Zhang H, et al. Endocardium Contributes to Cardiac Fat. Circ Res. 2016;118(2):254-265.

23. Smith CL, Baek ST, Sung CY, Tallquist MD. Epicardial-derived cell epithelial-to-mesenchymal transition and fate specification require PDGF receptor signaling. Circ Res. 2011;108(12):e15-e26.

24. Acharya A, et al. The bHLH transcription factor Tcf 21 is required for lineage-specific EMT of cardiac fibroblast progenitors. Development. 2012;139(12):2139-2149.

25. Song K, et al. Heart repair by reprogramming non-myocytes with cardiac transcription factors. Nature. 2012;485(7400):599-604.

26. Hsieh PC, et al. Evidence from a genetic fatemapping study that stem cells refresh adult mammalian cardiomyocytes after injury. Nat Med. 2007;13(8):970-974.

27. Wang Y, et al. Ephrin-B2 controls VEGF-induced angiogenesis and lymphangiogenesis. Nature. 2010;465(7297):483-486

28. Tian X, et al. Subepicardial endothelial cells invade the embryonic ventricle wall to form coronary arteries. Cell Res. 2013;23(9):1075-1090.

29. Imai T, Jiang M, Kastner P, Chambon P, Metzger D. Selective ablation of retinoid $X$ receptor alpha in hepatocytes impairs their lifespan and regenerative capacity. Proc Natl Acad Sci USA. 2001;98(8):4581-4586.

30. Sheikh AY, et al. In vivo genetic profiling and cel- lular localization of apelin reveals a hypoxia-sensitive, endothelial-centered pathway activated in ischemic heart failure. Am J Physiol Heart Circ Physiol. 2008;294(1):H88-H98.

31. Red-Horse K, Ueno H, Weissman IL, Krasnow MA. Coronary arteries form by developmental reprogramming of venous cells. Nature. 2010;464(7288):549-553.

32. Tian X, et al. Vessel formation. De novo formation of a distinct coronary vascular population in neonatal heart. Science. 2014;345(6192):90-94.

33. Liu Q, et al. Genetic targeting of sprouting angiogenesis using Apln-CreER. Nat Commun. 2015;6:6020.

34. Elmasri $H$, et al. Fatty acid binding protein 4 is a target of VEGF and a regulator of cell proliferation in endothelial cells. FASEB J. 2009;23(11):3865-3873.

35. Harjes U, Bridges E, McIntyre A, Fielding BA, Harris AL. Fatty acid-binding protein 4, a point of convergence for angiogenic and metabolic signaling pathways in endothelial cells. J Biol Chem. 2014;289(33):23168-23176.

36. He L, Tian X, Zhang H, Wythe JD, Zhou B. Fabp4-CreER lineage tracing reveals two distinctive coronary vascular populations. J Cell $\mathrm{Mol}$ Med. 2014;18(11):2152-2156.

37. Kong P, Christia P, Saxena A, Su Y, Frangogiannis NG. Lack of specificity of fibroblast-specific protein 1 in cardiac remodeling and fibrosis. Am JPhysiol Heart Circ Physiol. 2013;305(9):H1363-H1372.

38. Kretzschmar K, Watt FM. Lineage tracing. Cell. 2012;148(1-2):33-45.

39. Tian X, Pu WT, Zhou B. Cellular origin and developmental program of coronary angiogenesis. Circ Res. 2015;116(3):515-530.

40. van Berlo JH, et al. c-kit+ cells minimally contribute cardiomyocytes to the heart. Nature. 2014;509(7500):337-341.

41. Sultana N, et al. Resident c-kit(+) cells in the heart are not cardiac stem cells. Nat Commun. 2015;6:8701.

42. Liu Q, et al. Genetic lineage tracing identifies in situ Kit-expressing cardiomyocytes. Cell Res. 2016;26(1):119-130.

43. Oh H, et al. Cardiac progenitor cells from adult myocardium: homing, differentiation, and fusion after infarction. Proc Natl Acad Sci U S A. 2003;100(21):12313-12318.

44. Uchida S, et al. Sca1-derived cells are a source of 
myocardial renewal in the murine adult heart. Stem Cell Reports. 2013;1(5):397-410.

45. Zhou B, et al. Adult mouse epicardium modulates myocardial injury by secreting paracrine factors. JClin Invest. 2011;121(5):1894-1904.

46. Yamamoto $\mathrm{M}$, et al. A multifunctional reporter mouse line for Cre- and FLP-dependent lineage analysis. Genesis. 2009;47(2):107-114.
47. Furuyama K, et al. Continuous cell supply from a Sox9-expressing progenitor zone in adult liver, exocrine pancreas and intestine. Nat Genet. 2011;43(1):34-41.

48. Zhang $\mathrm{H}$, et al. Endocardium minimally contributes to coronary endothelium in the embryonic ventricular free walls. Circ Res. 2016;118(12):1880-1893.
49. Liu Q, et al. c-kit(+) cells adopt vascular endothelial but not epithelial cell fates during lung maintenance and repair. Nat Med. 2015;21(8):866-868.

50. Zhou B, von Gise A, Ma Q, Hu YW, Pu WT. Genetic fate mapping demonstrates contribution of epicardium-derived cells to the annulus fibrosis of the mammalian heart. Dev Biol. 2010;338(2):251-261. 